\title{
Novel Computational Methods for High-Dimensional Stochastic Sensitivity Analysis
}

\author{
Sharif Rahman*†, Xuchun Ren ${ }^{\ddagger}$ \\ College of Engineering, The University of Iowa, Iowa City, Iowa 52242, U.S.A.
}

\begin{abstract}
SUMMARY
This paper presents three new computational methods for calculating design sensitivities of statistical moments and reliability of high-dimensional complex systems subject to random input. The first method represents a novel integration of polynomial dimensional decomposition (PDD) of a multivariate stochastic response function and score functions. Applied to the statistical moments, the method provides mean-square convergent analytical expressions of design sensitivities of the first two moments of a stochastic response. The second and third methods, relevant to probability distribution or reliability analysis, exploit two distinct combinations built on PDD: the PDD-SPA method, entailing the saddlepoint approximation (SPA) and score functions; and the PDD-MCS method, utilizing the embedded Monte Carlo simulation (MCS) of the PDD approximation and score functions. For all three methods developed, the statistical moments or failure probabilities and their design sensitivities are both determined concurrently from a single stochastic analysis or simulation. Numerical examples, including a 100-dimensional mathematical problem, indicate that the new methods developed provide not only theoretically convergent or accurate design sensitivities, but also computationally efficient solutions. A practical example involving robust design optimization of a three-hole bracket illustrates the usefulness of the proposed methods. Copyright @ 2013 John Wiley \& Sons, Ltd.
\end{abstract}

Received ...

KEY WORDS: dimension reduction; orthogonal polynomials; polynomial dimensional decomposition; robust design optimization; saddlepoint approximation; score function

\section{INTRODUCTION}

Stochastic sensitivity analysis plays a central role in robust and reliability-based design optimizations (RDO and RBDO) of complex systems. For calculating design sensitivities of a stochastic response of interest, the finite-difference method [1] constitutes the most general and straightforward approach, but it mandates repeated stochastic analyses for different instances of design variables. Therefore, for practical design optimizations, the finite-difference method is very expensive, if not prohibitive. The two other prominent methods, the infinitesimal perturbation analysis [2] and the score function method [3], have been mostly viewed as competing methods, where both stochastic responses and sensitivities can be obtained from a single stochastic simulation. However, there are additional requirements of regularity conditions, in particular smoothness of the performance function or the probability measure. Both methods, when valid, are

${ }^{*}$ Correspondence to: Sharif Rahman, Department of Mechanical \& Industrial Engineering, The University of Iowa, Iowa City, Iowa 52242, U.S.A. E-mail: rahman@engineering.uiowa.edu

Contract/grant sponsor: U.S. National Science Foundation; contract/grant number: CMMI-0969044

${ }^{\dagger}$ Professor.

${ }^{\ddagger}$ Graduate student. 
typically employed in conjunction with crude Monte Carlo simulation (MCS). Unfortunately, for optimization of complex mechanical systems, where stochastic response and sensitivity analyses are required at each design iteration, even a single MCS is impractical, as each deterministic trial of simulation often requires expensive finite-element or other numerical calculations [4].

The dimensional decomposition is a finite, hierarchical, and convergent expansion of a multivariate output function in terms of its input variables with increasing dimensions [5]-8]. The decomposition ameliorates the curse of dimensionality [9] to some extent by developing an inputoutput behavior of complex systems with low effective dimensions [10], wherein the degrees of interactions between input variables attenuate rapidly or vanish altogether. Based on a coupling between dimensional decomposition and score function, Rahman [4] developed an efficient method for calculating design sensitivities of stochastic systems. The method, which sidesteps the need for crude MCS, is capable of estimating both the probabilistic response and its sensitivity from a single stochastic analysis without requiring performance function gradients. Another related method, proposed by Huang and Zhang [11], combines Daniel's saddlepoint approximation (SPA) [12] with Xu and Rahman's dimension-reduction integration technique [13] to perform stochastic sensitivity analysis. In their method, the sensitivity of reliability through SPA is connected to the sensitivities of moments of the performance function. To calculate the sensitivities of moments, the kernel functions, similar to the score functions, are used with dimension-reduction integration, which is the same as the dimensional decomposition exploited by Rahman [4]. Nonetheless, Huang and Zhang's method offers a few additional advantages: the tail probabilistic characteristics of a stochastic response, if they closely follow the exponential family of distributions, are accurately estimated by SPA; furthermore, the embedded MCS of Rahman [4] for calculating sensitivity of reliability is avoided. It is important to clarify that the "dimensional decomposition" and "dimension-reduction" concepts invoked by these two sensitivity methods are the same as the referential dimensional decomposition (RDD) formally presented in latter works [8 14]. Therefore, both methods essentially employ RDD for multivariate function approximations, where the mean values of random input are treated as the reference point [13]. The developments of these methods were motivated by the fact that RDD requires only function evaluations, as opposed to high-dimensional integrals required by another dimensional decomposition, known as the ANOVA dimensional decomposition [6] or its polynomial version, the polynomial dimensional decomposition (PDD) [15, 16]. However, a recent error analysis [8] reveals sub-optimality of RDD approximations, meaning that an RDD approximation, regardless of how the reference point is chosen, cannot be better than an ANOVA approximation for identical degrees of interaction. The analysis also finds ANOVA approximations to be exceedingly more precise than RDD approximations at higher-variate truncations. Therefore, a more precise function decomposition, such as the PDD [15, 16], which inherits all desirable properties of the ANOVA dimensional decomposition, should be employed for sensitivity analysis.

This paper presents three new computational methods for calculating design sensitivities of statistical moments and reliability of high-dimensional complex systems subject to random input. The first method represents a novel integration of PDD of a multivariate stochastic response function and Fourier-polynomial expansions of score functions associated with the probability measure of the random input. Applied to the statistical moments, the method provides analytical expressions of design sensitivities of the first two moments of a stochastic response. The second and third methods, relevant to probability distribution or reliability analysis, exploit two distinct combinations grounded in PDD: the PDD-SPA method, entailing SPA and score functions; and the PDD-MCS method, utilizing the embedded MCS of PDD approximation and score functions. Section 2 describes the PDD approximation of a multivariate function, resulting in explicit formulae for the first two moments, and the PDD-SPA and PDD-MCS methods for reliability analysis. Section 3 defines score functions and unveils new closed-form formulae or numerical procedures for design sensitivities of moments. The convergence of the sensitivities of moments by the proposed method is also proved in this section. Section 4 describes the PDD-SPA and PDD-MCS methods for sensitivity analysis and explains how the effort required to calculate the failure probability also delivers its design sensitivities, sustaining no additional cost. The calculation of PDD expansion coefficients, required in sensitivity analyses of both moments and failure probability, is discussed in Section 5. In 
Section 6, six numerical examples are presented to probe the convergence properties, accuracy, and computational efficiency of the proposed methods, including design optimization of a three-hole bracket. Finally, conclusions are drawn in Section 7.

\section{POLYNOMIAL DIMENSIONAL DECOMPOSITION METHODS FOR STOCHASTIC ANALYSES}

Let $\mathbb{N}, \mathbb{N}_{0}, \mathbb{R}$, and $\mathbb{R}_{0}^{+}$represent the sets of positive integer (natural), non-negative integer, real, and non-negative real numbers, respectively. For $k \in \mathbb{N}$, denote by $\mathbb{R}^{k}$ the $k$-dimensional Euclidean space and by $\mathbb{N}_{0}^{k}$ the $k$-dimensional multi-index space. These standard notations will be used throughout the paper.

Consider a measurable space $(\Omega, \mathcal{F})$, where $\Omega$ is a sample space and $\mathcal{F}$ is a $\sigma$-field on $\Omega$. Defined over $(\Omega, \mathcal{F})$, let $\left\{P_{\mathbf{d}}: \mathcal{F} \rightarrow[0,1]\right\}$ be a family of probability measures, where for $M \in \mathbb{N}$ and $N \in \mathbb{N}, \mathbf{d}=\left(d_{1}, \cdots, d_{M}\right) \in \mathcal{D}$ is an $\mathbb{R}^{M}$-valued design vector with non-empty closed set $\mathcal{D} \subseteq \mathbb{R}^{M}$, and $\mathbf{X}:=\left(X_{1}, \cdots, X_{N}\right):(\Omega, \mathcal{F}) \rightarrow\left(\mathbb{R}^{N}, \mathcal{B}^{N}\right)$ be an $\mathbb{R}^{N}$-valued input random vector with $\mathcal{B}^{N}$ representing the Borel $\sigma$-field on $\mathbb{R}^{N}$, describing the statistical uncertainties in loads, material properties, and the geometry of a complex mechanical system. The probability law of $\mathbf{X}$ is completely defined by a family of the joint probability density functions (PDF) $\left\{f_{\mathbf{X}}(\mathbf{x} ; \mathbf{d}), \mathbf{x} \in\right.$ $\left.\mathbb{R}^{N}, \mathbf{d} \in \mathcal{D}\right\}$ that are associated with probability measures $\left\{P_{\mathbf{d}}, \mathbf{d} \in \mathcal{D}\right\}$, so that the probability triple $\left(\Omega, \mathcal{F}, P_{\mathbf{d}}\right)$ of $\mathbf{X}$ depends on $\mathbf{d}$. A design variable $d_{k}$ can be any distribution parameter or a statistic - for instance, the mean or standard deviation - of one or more random variables.

\subsection{Polynomial dimensional decomposition}

Let $y(\mathbf{X})$ be a real-valued, square-integrable, measurable transformation on $(\Omega, \mathcal{F})$, describing the relevant performance function of a complex system. It is assumed that $y:\left(\mathbb{R}^{N}, \mathcal{B}^{N}\right) \rightarrow(\mathbb{R}, \mathcal{B})$ is not an explicit function of $\mathbf{d}$, although $y$ implicitly depends on $\mathbf{d}$ via the probability law of $\mathbf{X}$. Assuming independent coordinates of $\mathbf{X}$, its joint PDF is expressed by a product, $f_{\mathbf{X}}(\mathbf{x} ; \mathbf{d})=$ $\prod_{i=1}^{i=N} f_{X_{i}}\left(x_{i} ; \mathbf{d}\right)$, of marginal PDF $f_{X_{i}}: \mathbb{R} \rightarrow \mathbb{R}_{0}^{+}$of $X_{i}, i=1, \cdots, N$, defined on its probability triple $\left(\Omega_{i}, \mathcal{F}_{i}, P_{i, \mathbf{d}}\right)$ with a bounded or an unbounded support on $\mathbb{R}$. Then, for a given subset $u \subseteq\{1, \cdots, N\}, f_{\mathbf{X}_{-u}}\left(\mathbf{x}_{-u} ; \mathbf{d}\right):=\prod_{i=1, i \notin u}^{N} f_{X_{i}}\left(x_{i} ; \mathbf{d}\right)$ defines the marginal density function of $\mathbf{X}_{-u}:=\mathbf{X}_{\{1, \cdots, N\} \backslash u}$.

2.1.1. ANOVA dimensional decomposition The analysis-of-variance (ANOVA) dimensional decomposition, expressed by the recursive form [6-8]

$$
\begin{aligned}
y(\mathbf{X}) & =\sum_{u \subseteq\{1, \cdots, N\}} y_{u}\left(\mathbf{X}_{u} ; \mathbf{d}\right), \\
y_{\emptyset}(\mathbf{d}) & =\int_{\mathbb{R}^{N}} y(\mathbf{x}) f_{\mathbf{X}}(\mathbf{x} ; \mathbf{d}) d \mathbf{x}, \\
y_{u}\left(\mathbf{X}_{u} ; \mathbf{d}\right) & =\int_{\mathbb{R}^{N-|u|}} y\left(\mathbf{X}_{u}, \mathbf{x}_{-u}\right) f_{\mathbf{X}_{-u}}\left(\mathbf{x}_{-u} ; \mathbf{d}\right) d \mathbf{x}_{-u}-\sum_{v \subset u} y_{v}\left(\mathbf{X}_{v} ; \mathbf{d}\right),
\end{aligned}
$$

is a finite, hierarchical expansion of $y$ in terms of its input variables with increasing dimensions, where $u \subseteq\{1, \cdots, N\}$ is a subset with the complementary set $-u=\{1, \cdots, N\} \backslash u$ and cardinality $0 \leq|u| \leq N$, and $y_{u}$ is a $|u|$-variate component function describing the interactive effect of $\mathbf{X}_{u}=\left(X_{i_{1}}, \cdots, X_{i_{|u|}}\right), 1 \leq i_{1}<\cdots<i_{|u|} \leq N$, a subvector of $\mathbf{X}$. The summation in Equation 1 comprises $2^{N}$ terms, with each term depending on a group of variables indexed by a particular subset of $\{1, \cdots, N\}$, including the empty set $\emptyset$.

The ANOVA component functions $y_{u}, \emptyset \neq u \subseteq\{1, \cdots, N\}$, have two remarkable properties: (1) the component functions, $y_{u}, \emptyset \neq u \subseteq\{1, \cdots, N\}$, have zero means; and (2) any two distinct component functions $y_{u}$ and $y_{v}$, where $u \subseteq\{1, \cdots, N\}, v \subseteq\{1, \cdots, N\}$, and $u \neq v$, are orthogonal. Further details are available elsewhere [8]. 
Remark 1. The coefficient $y_{\emptyset}=\mathbb{E}_{\mathbf{d}}[y(\mathbf{X})]$ in Equation 2 is a function of the design vector $\mathbf{d}$, which describes the probability distribution of the random vector $\mathbf{X}$. Therefore, the adjective "constant" used to describe $y_{\emptyset}$ should be interpreted with respect to $\mathbf{X}$, not $\mathbf{d}$. A similar condition applies for the non-constant component functions $y_{u}, \emptyset \neq u \subseteq\{1, \cdots, N\}$, which also depend on $\mathbf{d}$.

2.1.2. Orthonormal Polynomials and Stochastic Expansions Let $\left\{\psi_{i j}\left(x_{i} ; \mathbf{d}\right) ; j=0,1, \cdots\right\}$ be a set of univariate, orthonormal polynomial basis functions in the Hilbert space $\mathcal{L}_{2}\left(\Omega_{i}, \mathcal{F}_{i}, P_{i, \mathbf{d}}\right)$ that is consistent with the probability measure $P_{i, \mathbf{d}}$ or $f_{X_{i}}\left(x_{i} ; \mathbf{d}\right) d x_{i}$ of $X_{i}$ for a given design $\mathbf{d}$. For $\emptyset \neq u=\left\{i_{1}, \cdots, i_{|u|}\right\} \subseteq\{1, \cdots, N\}$, where $1 \leq|u| \leq N$, let $\left(\times_{p=1}^{p=|u|} \Omega_{i_{p}}, \times_{p=1}^{p=|u|} \mathcal{F}_{i_{p}}, \times_{p=1}^{p=|u|} P_{i_{p}, \mathbf{d}}\right)$ be the product probability triple of $\mathbf{X}_{u}=\left(X_{i_{1}}, \cdots, X_{i_{|u|}}\right)$. Denote the associated space of the $|u|$ variate component functions of $y$ by

$$
\mathcal{L}_{2}\left(\times_{p=1}^{p=|u|} \Omega_{i_{p}}, \times_{p=1}^{p=|u|} \mathcal{F}_{i_{p}}, \times_{p=1}^{p=|u|} P_{i_{p}, \mathbf{d}}\right):=\left\{y_{u}: \int_{\mathbb{R}^{|u|}} y_{u}^{2}\left(\mathbf{x}_{u} ; \mathbf{d}\right) f_{\mathbf{X}_{u}}\left(\mathbf{x}_{u} ; \mathbf{d}\right) d \mathbf{x}_{u}<\infty\right\},
$$

which is a Hilbert space. Since the joint density of $\mathbf{X}_{u}$ is separable (independence of $X_{i}, i \in u$ ), that is, $f_{\mathbf{X}_{u}}\left(\mathbf{x}_{u} ; \mathbf{d}\right)=\prod_{p=1}^{|u|} f_{X_{i_{p}}}\left(x_{i_{p}} ; \mathbf{d}\right)$, the product $\psi_{u \mathbf{j}_{|u|}}\left(\mathbf{X}_{u} ; \mathbf{d}\right):=\prod_{p=1}^{|u|} \psi_{i_{p} j_{p}}\left(X_{i_{p}} ; \mathbf{d}\right)$, where $\mathbf{j}_{|u|}=\left(j_{1}, \cdots, j_{|u|}\right) \in \mathbb{N}_{0}^{|u|}$, a $|u|$-dimensional multi-index, constitutes a multivariate orthonormal polynomial basis in $\mathcal{L}_{2}\left(\times_{p=1}^{p=|u|} \Omega_{i_{p}}, \times_{p=1}^{p=|u|} \mathcal{F}_{i_{p}}, \times_{p=1}^{p=|u|} P_{i_{p}, \mathbf{d}}\right)$. Two important properties of these product polynomials from tensor products of Hilbert spaces are as follows.

Proposition 2. The product polynomials $\psi_{u \mathbf{j}|u|}\left(\mathbf{X}_{u} ; \mathbf{d}\right), \emptyset \neq u \subseteq\{1, \cdots, N\}, j_{1}, \cdots, j_{|u|} \neq 0, \mathbf{d} \in$ $\mathcal{D}$, have zero means, i.e.,

$$
\mathbb{E}_{\mathbf{d}}\left[\psi_{u \mathbf{j}_{|u|}}\left(\mathbf{X}_{u} ; \mathbf{d}\right)\right]=0 \text {. }
$$

Proposition 3. Any two distinct product polynomials $\psi_{u \mathbf{j}_{|u|}}\left(\mathbf{X}_{u} ; \mathbf{d}\right)$ and $\psi_{v \mathbf{k}_{|v|}}\left(\mathbf{X}_{v} ; \mathbf{d}\right)$ for $\mathbf{d} \in \mathcal{D}$, where $\emptyset \neq u \subseteq\{1, \cdots, N\}, \emptyset \neq v \subseteq\{1, \cdots, N\}, j_{1}, \cdots, j_{|u|} \neq 0, k_{1}, \cdots, k_{|v|} \neq 0$, are uncorrelated and each has unit variance, i.e.,

$$
\mathbb{E}_{\mathbf{d}}\left[\psi_{u \mathbf{j}|u|}\left(\mathbf{X}_{u} ; \mathbf{d}\right) \psi_{v \mathbf{k}_{|v|}}\left(\mathbf{X}_{v} ; \mathbf{d}\right)\right]= \begin{cases}1 & \text { if } u=v ; \mathbf{j}_{|u|}=\mathbf{k}_{|v|} \\ 0 & \text { otherwise. }\end{cases}
$$

Remark 4. Given a probability measure $P_{i, \mathbf{d}}$ of any random variable $X_{i}$, the well-known three-term recurrence relation is commonly used to construct the associated orthogonal polynomials [16, 17]. For $m \in \mathbb{N}$, the first $m$ recursion coefficient pairs are uniquely determined by the first $2 m$ moments of $X_{i}$ that must exist. When these moments are exactly calculated, they lead to exact recursion coefficients, some of which belong to classical orthogonal polynomials. For an arbitrary probability measure, approximate methods, such as the Stieltjes procedure, can be employed to obtain the recursion coefficients [16, 17].

The orthogonal polynomial expansion of a non-constant $|u|$-variate ANOVA component function in Equation 3 becomes [15, 16]

$$
y_{u}\left(\mathbf{X}_{u} ; \mathbf{d}\right)=\sum_{\substack{\mathbf{j}_{|u|} \in \mathbb{N}_{0}^{|u|} \\ j_{1}, \cdots, j_{|u|} \neq 0}} C_{u \mathbf{j}_{|u|}}(\mathbf{d}) \psi_{u \mathbf{j}_{|u|}}\left(\mathbf{X}_{u} ; \mathbf{d}\right)
$$

for any $\emptyset \neq u \subseteq\{1, \cdots, N\}$ with

$$
C_{u \mathbf{j}_{|u|}}(\mathbf{d}):=\int_{\mathbb{R}^{N}} y(\mathbf{x}) \psi_{u \mathbf{j}_{|u|}}\left(\mathbf{x}_{u} ; \mathbf{d}\right) f_{\mathbf{X}}(\mathbf{x} ; \mathbf{d}) d \mathbf{x}
$$

representing the corresponding expansion coefficient. Similar to $y_{\emptyset}$, the coefficient $C_{u \mathbf{j}_{|u|}}$ also depends on the design vector $\mathbf{d}$. When $u=\{i\}, i=1, \cdots, N$, the univariate component functions and expansion coefficients are

$$
y_{\{i\}}\left(X_{i} ; \mathbf{d}\right)=\sum_{j=1}^{\infty} C_{i j}(\mathbf{d}) \psi_{i j}\left(X_{i} ; \mathbf{d}\right)
$$


and $C_{i j}(\mathbf{d}):=C_{\{i\}(j)}(\mathbf{d})$, respectively. When $u=\left\{i_{1}, i_{2}\right\}, i_{1}=1, \cdots, N-1, i_{2}=i_{1}+1, \cdots, N$, the bivariate component functions and expansion coefficients are

$$
y_{\left\{i_{1}, i_{2}\right\}}\left(X_{i_{1}}, X_{i_{2}} ; \mathbf{d}\right)=\sum_{j_{1}=1}^{\infty} \sum_{j_{2}=1}^{\infty} C_{i_{1} i_{2} j_{1} j_{2}}(\mathbf{d}) \psi_{i_{1} j_{1}}\left(X_{i_{1}} ; \mathbf{d}\right) \psi_{i_{2} j_{2}}\left(X_{i_{2}} ; \mathbf{d}\right)
$$

and $C_{i_{1} i_{2} j_{1} j_{2}}(\mathbf{d}):=C_{\left\{i_{1}, i_{2}\right\}\left(j_{1}, j_{2}\right)}(\mathbf{d})$, respectively, and so on. Using Propositions 2 and 3 , all component functions $y_{u}, \emptyset \neq u \subseteq\{1, \cdots, N\}$, are found to satisfy the annihilating conditions of the ANOVA dimensional decomposition. The end result of combining Equations 13 and 7 is the PDD [15, 16],

$$
y(\mathbf{X})=y_{\emptyset}(\mathbf{d})+\sum_{\emptyset \neq u \subseteq\{1, \cdots, N\}} \sum_{\substack{\mathbf{j}_{|u|} \in \mathbb{N}_{0}^{|u|} \\ j_{1}, \cdots, j_{|u|} \neq 0}} C_{u \mathbf{j}_{|u|}}(\mathbf{d}) \psi_{u \mathbf{j}|u|}\left(\mathbf{X}_{u} ; \mathbf{d}\right),
$$

providing a hierarchical expansion of $y$ in terms of an infinite number of coefficients and orthonormal polynomials. In practice, the number of coefficients or polynomials must be finite, say, by retaining at most $m$ th-order polynomials in each variable. Furthermore, in many applications, the function $y$ can be approximated by a sum of at most $S$-variate component functions, where $S \in \mathbb{N}$; $1 \leq S \leq N$, resulting in the $S$-variate, $m$ th-order PDD approximation

$$
\tilde{y}_{S, m}(\mathbf{X})=y_{\emptyset}(\mathbf{d})+\sum_{\substack{\emptyset \neq u \subseteq\{1, \cdots, N\} \\ 1 \leq|u| \leq S}} \sum_{\substack{\mathbf{j}_{|u|} \in \mathbb{N}_{0}^{|u|},\left\|\mathbf{j}_{|u|}\right\|_{\infty} \leq m \\ j_{1}, \cdots, j_{|u|} \neq 0}} C_{u \mathbf{j}_{|u|}}(\mathbf{d}) \psi_{u \mathbf{j}|u|}\left(\mathbf{X}_{u} ; \mathbf{d}\right),
$$

containing $\sum_{k=0}^{S}\left(\begin{array}{c}N \\ k\end{array}\right) m^{k}$ number of PDD coefficients and corresponding orthonormal polynomials. The inner sum of Equation 12 contains the $\infty$-norm $\left\|\mathbf{j}_{|u|}\right\|_{\infty}:=\max \left(j_{1}, \cdots, j_{|u|}\right) \in \mathbb{N}_{0}^{|u|}$ and precludes $j_{1}, \cdots, j_{|u|} \neq 0$, that is, the individual degree of each variable $X_{i}$ in $\psi_{u \mathbf{j}}, i \in \mid, u$, can not be zero since $y_{u}$ is a zero-mean strictly $|u|$-variate function. Due to its additive structure, the approximation in Equation 12 includes degrees of interaction among at most $S$ input variables $X_{i_{1}}, \cdots, X_{i_{S}}, 1 \leq i_{1} \leq \cdots \leq i_{S} \leq N$. For instance, by selecting $S=1$ and 2 , the functions

$$
\tilde{y}_{1, m}(\mathbf{X})=y_{\emptyset}+\sum_{i=1}^{N} \sum_{j=1}^{m} C_{i j}(\mathbf{d}) \psi_{i j}\left(X_{i} ; \mathbf{d}\right)
$$

and

$$
\begin{aligned}
\tilde{y}_{2, m}(\mathbf{X})= & y_{\emptyset}(\mathbf{d})+\sum_{i=1}^{N} \sum_{j=1}^{m} C_{i j}(\mathbf{d}) \psi_{i j}\left(X_{i} ; \mathbf{d}\right)+ \\
& \sum_{i_{1}=1}^{N-1} \sum_{i_{2}=i_{1}+1}^{N} \sum_{j_{1}=1}^{m} \sum_{j_{2}=1}^{m} C_{i_{1} i_{2} j_{1} j_{2}}(\mathbf{d}) \psi_{i_{1} j_{1}}\left(X_{i_{1}} ; \mathbf{d}\right) \psi_{i_{2} j_{2}}\left(X_{i_{2}} ; \mathbf{d}\right),
\end{aligned}
$$

respectively, provide univariate and bivariate $m$ th-order PDD approximations, contain contributions from all input variables, and should not be viewed as first- and second-order approximations, nor as limiting the nonlinearity of $y$. Depending on how the component functions are constructed, arbitrarily high-order univariate and bivariate terms of $y$ could be lurking inside $\tilde{y}_{1, m}$ and $\tilde{y}_{2, m}$. When $S \rightarrow N$ and $m \rightarrow \infty, \tilde{y}_{S, m}$ converges to $y$ in the mean-square sense, permitting Equation 12 to generate a hierarchical and convergent sequence of approximations of $y$. Readers interested in further details of PDD are referred to the authors' past works [15, 16].

\subsection{Statistical Moment Analysis}

Let $m^{(r)}(\mathbf{d}):=\mathbb{E}_{\mathbf{d}}\left[y^{r}(\mathbf{X})\right]$, if it exists, define the raw moment of $y$ of order $r$, where $r \in \mathbb{N}$. Given an $S$-variate, $m$ th-order PDD approximation $\tilde{y}_{S, m}(\mathbf{X})$ of $y(\mathbf{X})$, let $\tilde{m}_{S, m}^{(r)}(\mathbf{d}):=\mathbb{E}_{\mathbf{d}}\left[\tilde{y}_{S, m}^{r}(\mathbf{X})\right]$ define the raw moment of $\tilde{y}_{S, m}$ of order $r$. The following subsections describe the explicit formulae or analytical expressions for calculating the moments by PDD approximations. 
2.2.1. First- and Second-Order Moments Applying the expectation operator on $\tilde{y}_{S, m}(\mathbf{X})$ and $\tilde{y}_{S, m}^{2}(\mathbf{X})$, and recognizing Propositions 2 and 3 the first moment or mean [18]

$$
\tilde{m}_{S, m}^{(1)}(\mathbf{d}):=\mathbb{E}_{\mathbf{d}}\left[\tilde{y}_{S, m}(\mathbf{X})\right]=y_{\emptyset}(\mathbf{d})=\mathbb{E}_{\mathbf{d}}[y(\mathbf{X})]=: m^{(1)}(\mathbf{d})
$$

of the $S$-variate, $m$ th-order PDD approximation matches the exact mean of $y$, regardless of $S$ or $m$, whereas the second moment [18]

$$
\tilde{m}_{S, m}^{(2)}(\mathbf{d}):=\mathbb{E}_{\mathbf{d}}\left[\tilde{y}_{S, m}^{2}(\mathbf{X})\right]=y_{\emptyset}^{2}(\mathbf{d})+\sum_{\substack{\emptyset \neq u \subseteq\{1, \cdots, N\} \\ 1 \leq|u| \leq S}} \sum_{\substack{\mathbf{j}_{|u|} \in \mathbb{N}_{0}^{|u|},|| \mathbf{j}_{|u|} \mid \|_{\infty} \leq m \\ j_{1}, \cdots, j_{|u|} \neq 0}} C_{u \mathbf{j}_{|u|}}^{2}(\mathbf{d})
$$

is calculated as the sum of squares of all expansion coefficients of $\tilde{y}_{S, m}(\mathbf{X})$. Clearly, the approximate second moment in Equation 16 approaches the exact second moment

$$
m^{(2)}(\mathbf{d}):=\mathbb{E}_{\mathbf{d}}\left[y^{2}(\mathbf{X})\right]=y_{\emptyset}^{2}(\mathbf{d})+\sum_{\emptyset \neq u \subseteq\{1, \cdots, N\}} \sum_{\substack{\mathbf{j}_{|u|} \in \mathbb{N}_{0}^{|u|} \\ j_{1}, \cdots, j_{|u|} \neq 0}} C_{u \mathbf{j}_{|u|}}^{2}(\mathbf{d})
$$

of $y$ when $S \rightarrow N$ and $m \rightarrow \infty$. The mean-square convergence of $\tilde{y}_{S, m}$ is guaranteed as $y$, and its component functions are all members of the associated Hilbert spaces. In addition, the variance of $\tilde{y}_{S, m}(\mathbf{X})$ is also mean-square convergent.

For the two special cases, $S=1$ and $S=2$, the univariate and bivariate PDD approximations yield the same exact mean value $y_{\emptyset}(\mathbf{d})$, as noted in Equation 15 . However, the respective second moment approximations,

$$
\tilde{m}_{1, m}^{(2)}(\mathbf{d})=y_{\emptyset}^{2}(\mathbf{d})+\sum_{i=1}^{N} \sum_{j=1}^{m} C_{i j}^{2}(\mathbf{d})
$$

and

$$
\tilde{m}_{2, m}^{(2)}(\mathbf{d})=y_{\emptyset}^{2}(\mathbf{d})+\sum_{i=1}^{N} \sum_{j=1}^{m} C_{i j}^{2}(\mathbf{d})+\sum_{i_{1}=1}^{N-1} \sum_{i_{2}=i_{1}+1}^{N} \sum_{j_{2}=1}^{m} \sum_{j_{1}=1}^{m} C_{i_{1} i_{2} j_{1} j_{2}}^{2}(\mathbf{d}),
$$

differ, depend on $m$, and progressively improve as $S$ becomes larger. Recent works on error analysis indicate that the second-moment properties obtained from the ANOVA dimensional decomposition, which leads to PDD approximations, are superior to those derived from dimension-reduction methods that are grounded in RDD [8, 14].

2.2.2. Higher-Order Moments When calculating higher-order $(2<r<\infty)$ moments by the PDD approximation, no explicit formulae exist for a general function $y$ or the probability distribution of $\mathbf{X}$. In which instance, two options are proposed to estimate the higher-order moments.

Option I entails expanding the $r$ th power of the PDD approximation of $y$ by

$$
\tilde{y}_{S, m}^{r}(\mathbf{X})=g_{\emptyset}(\mathbf{d})+\sum_{\substack{\emptyset \neq u \subseteq\{1, \cdots, N\} \\ 1 \leq|u| \leq \min (r S, N)}} g_{u}\left(\mathbf{X}_{u} ; \mathbf{d}\right)
$$

in terms of a constant $g_{\emptyset}(\mathbf{d})$ and at $\operatorname{most} \min (r S, N)$-variate polynomial functions $g_{u}\left(\mathbf{X}_{u} ; \mathbf{d}\right)$ and then calculating the moment

$$
\begin{aligned}
\tilde{m}_{S, m}^{(r)}(\mathbf{d}) & =\int_{\mathbb{R}^{N}} \tilde{y}_{S, m}^{r}(\mathbf{x}) f_{\mathbf{X}}(\mathbf{x} ; \mathbf{d}) d \mathbf{x} \\
& =g_{\emptyset}(\mathbf{d})+\sum_{\substack{\emptyset \neq u \subseteq\{1, \cdots, N\} \\
1 \leq|u| \leq \min (r S, N)}} \int_{\mathbb{R}^{|u|}} g_{u}\left(\mathbf{x}_{u} ; \mathbf{d}\right) f_{\mathbf{X}_{u}}\left(\mathbf{x}_{u} ; \mathbf{d}\right) d \mathbf{x}_{u}
\end{aligned}
$$

by integration, if it exists. For well-behaved functions, including many encountered in practical applications, $\tilde{m}_{S, m}^{(r)}(\mathbf{d})$ should render an accurate approximation of $m^{(r)}(\mathbf{d})$, the $r$ th-order moment 
of $y(\mathbf{X})$, although there is no rigorous mathematical proof of convergence when $r>2$. Note that Equation 21 involves integrations of elementary polynomial functions and does not require any expensive evaluation of the original function $y$. Nonetheless, since $\tilde{y}_{S, m}(\mathbf{X})$ is a superposition of at most $S$-variate component functions of independent variables, the largest dimension of the integrals in Equation 21 is $\min (r S, N)$. Therefore, Option I mandates high-dimensional integrations if $\min (r S, N)$ is large. In addition, if $r S \geq N$ and $N$ is large, then the resulting $N$-dimensional integration is infeasible.

As an alternative, Option II, relevant to large $N$, creates an additional $\bar{S}$-variate, $\bar{m}$ th-order PDD approximation

$$
\tilde{z}_{\bar{S}, \bar{m}}(\mathbf{X})=z_{\emptyset}(\mathbf{d})+\sum_{\substack{\emptyset \neq u \subseteq\{1, \cdots, N\} \\ 1 \leq|u| \leq \bar{S}}} \sum_{\substack{\mathbf{j}_{|u|} \in \mathbb{N}_{0}^{|u|},\left\|\mathbf{j}_{|u|}\right\|_{\infty} \leq \bar{m} \\ j_{1}, \cdots, j_{|u|} \neq 0}} \bar{C}_{u \mathbf{j}_{|u|}}(\mathbf{d}) \psi_{u \mathbf{j}_{|u|}}\left(\mathbf{X}_{u} ; \mathbf{d}\right)
$$

of $\tilde{y}_{S, m}^{r}(\mathbf{X})$, where $\bar{S}$ and $\bar{m}$, potentially distinct from $S$ and $m$, are accompanying truncation parameters, $z_{\emptyset}(\mathbf{d}):=\int_{\mathbb{R}^{N}} \tilde{y}_{S, m}^{r}(\mathbf{x}) f_{\mathbf{X}}(\mathbf{x} ; \mathbf{d}) d \mathbf{x}$, and $\bar{C}_{u \mathbf{j}_{|u|}}(\mathbf{d}):=\int_{\mathbb{R}^{N}} \tilde{y}_{S, m}^{r}(\mathbf{x}) \psi_{u \mathbf{j}_{|u|}}\left(\mathbf{x}_{u} ; \mathbf{d}\right) f_{\mathbf{X}}(\mathbf{x} ; \mathbf{d}) d \mathbf{x}$ are the associated PDD expansion coefficients of $\tilde{z}_{\bar{S}, \bar{m}}(\mathbf{X})$. Replacing $\tilde{y}_{S, m}^{r}(\mathbf{x})$ with $\tilde{z}_{\bar{S}, \bar{m}}(\mathbf{x})$, the first line of Equation 21 produces

$$
\tilde{m}_{S, m}^{(r)}(\mathbf{d})=\int_{\mathbb{R}^{N}} \tilde{z}_{\bar{S}, \bar{m}}(\mathbf{x}) f_{\mathbf{X}}(\mathbf{x} ; \mathbf{d}) d \mathbf{x}=: z_{\emptyset}(\mathbf{d}) .
$$

Then the evaluation of $z_{\emptyset}(\mathbf{d})$ from the definition, which also requires $N$-dimensional integration, leads Equation 23 back to Equation 21, raising the question of why Option II is introduced. Indeed, the distinction between the two options forms when the constant $z_{\emptyset}(\mathbf{d})$ is approximately calculated by dimension-reduction integration, to be explained in Section 5, entailing at most $\bar{S}$-dimensional integrations. Nonetheless, if $\bar{S} \ll r S<N$, then a significant dimension reduction is possible in Option II for estimating higher-order moments. In other words, Option II, which is an approximate version of Option I, may provide efficient solutions to high-dimensional problems, provided that a loss of accuracy in Option II, if any, is insignificant. The higher-order moments are useful for approximating the probability distribution of a stochastic response or reliability analysis, including their sensitivity analyses, and will be revisited in the next subsection.

\subsection{Reliability Analysis}

A fundamental problem in reliability analysis entails calculation of the failure probability

$$
P_{F}(\mathbf{d}):=P_{\mathbf{d}}\left[\mathbf{X} \in \Omega_{F}\right]=\int_{\mathbb{R}^{N}} I_{\Omega_{F}}(\mathbf{x}) f_{\mathbf{X}}(\mathbf{x} ; \mathbf{d}) d \mathbf{x}=: \mathbb{E}_{\mathbf{d}}\left[I_{\Omega_{F}}(\mathbf{X})\right]
$$

where $\Omega_{F}$ is the failure set and $I_{\Omega_{F}}(\mathbf{x})$ is the associated indicator function, which is equal to one when $\mathrm{x} \in \Omega_{F}$ and zero otherwise. Depending on the nature of the failure domain $\Omega_{F}$, a component or a system reliability analysis can be envisioned. For component reliability analysis, the failure domain is often adequately described by a single performance function $y(\mathbf{x})$, for instance, $\Omega_{F}:=$ $\{\mathbf{x}: y(\mathbf{x})<0\}$. In contrast, multiple, interdependent performance functions $y_{i}(\mathbf{x}), i=1,2, \cdots$, are required for system reliability analysis, leading, for example, to $\Omega_{F}:=\left\{\mathbf{x}: \cup_{i} y_{i}(\mathbf{x})<0\right\}$ and $\Omega_{F}:=\left\{\mathbf{x}: \cap_{i} y_{i}(\mathbf{x})<0\right\}$ for series and parallel systems, respectively. In this subsection, two methods are presented for estimating the failure probability. The PDD-SPA method, which blends the PDD approximation with SPA, is described first. Then the PDD-MCS method, which exploits the PDD approximation for MCS, is elucidated.

2.3.1. The PDD-SPA Method Let $F_{y}(\xi):=P_{\mathbf{d}}[y \leq \xi]$ be the cumulative distribution function (CDF) of $y(\mathbf{X})$. Assume that the PDF $f_{y}(\xi):=d F_{y}(\xi) / d \xi$ exists and suppose that the cumulant generating function (CGF)

$$
K_{y}(t):=\ln \left\{\int_{-\infty}^{+\infty} \exp (t \xi) f_{y}(\xi) d \xi\right\}
$$


of $y$ converges for $t \in \mathbb{R}$ in some non-vanishing interval containing the origin. Using inverse Fourier transformation, exponential power series expansion, and Hermite polynomial approximation, Daniels [12] developed an SPA formula to approximately evaluate $f_{y}(\xi)$. However, the success of such formula is predicated on how accurately the CGF and its derivatives, if they exist, are calculated. In fact, determining $K_{y}(t)$ is immensely difficult because it is equivalent to knowing all higher-order moments of $y$. To mitigate this problem, consider the Taylor series expansion of

$$
K_{y}(t)=\sum_{r \in \mathbb{N}} \frac{\kappa^{(r)} t^{r}}{r !}
$$

at $t=0$, where $\kappa^{(r)}:=d^{r} K_{y}(0) / d t^{r}, r \in \mathbb{N}$, is known as the $r$ th-order cumulant of $y(\mathbf{X})$. If some of these cumulants are effectively estimated, then a truncated Taylor series provides a useful means to approximate $K_{y}(t)$. For instance, assume that, given a positive integer $Q<\infty$, the raw moments $\tilde{m}_{S, m}^{(r)}(\mathbf{d})$ of order at most $Q$ have been calculated with sufficient accuracy using an $S$-variate, $m$ thorder PDD approximation $\tilde{y}_{S, m}(\mathbf{X})$ of $y(\mathbf{X})$, as described in the preceding subsection. Then the corresponding approximate cumulants are easily obtained from the well-known cumulant-moment relationship,

$$
\tilde{\kappa}_{S, m}^{(r)}(\mathbf{d})= \begin{cases}\tilde{m}_{S, m}^{(1)}(\mathbf{d}) & : \quad r=1, \\
\tilde{m}_{S, m}^{(r)}(\mathbf{d})-\sum_{p=1}^{r-1}\left(\begin{array}{l}
r-1 \\
p-1
\end{array}\right) \tilde{\kappa}_{S, m}^{(p)}(\mathbf{d}) \tilde{m}_{S, m}^{(r-p)}(\mathbf{d}) & : \quad 2 \leq r \leq Q,\end{cases}
$$

where the functional argument $\mathbf{d}$ serves as a reminder that the moments and cumulants all depend on the design vector d. Setting $\kappa^{(r)}=\tilde{\kappa}_{S, m}^{(r)}$ for $r=1, \cdots, Q$, and zero otherwise in Equation 26 , the result is an $S$-variate, $m$ th-order PDD approximation

$$
\tilde{K}_{y, Q, S, m}(t ; \mathbf{d})=\sum_{r=1}^{Q} \frac{\tilde{\kappa}_{S, m}^{(r)}(\mathbf{d}) t^{r}}{r !}
$$

of the $Q$ th-order Taylor series expansion of $K_{y}(t)$. It is elementary to show that $\tilde{K}_{y, Q, S, m}(t ; \mathbf{d}) \rightarrow$ $K_{y}(t)$ when $S \rightarrow N, m \rightarrow \infty$, and $Q \rightarrow \infty$.

Using the CGF approximation in Equation 28, Daniel's SPA leads to the explicit formula [12],

$$
\tilde{f}_{y, P S}(\xi ; \mathbf{d})=\left[2 \pi \tilde{K}^{\prime \prime}{ }_{y, Q, S, m}\left(t_{s} ; \mathbf{d}\right)\right]^{-\frac{1}{2}} \exp \left[\tilde{K}_{y, Q, S, m}\left(t_{s} ; \mathbf{d}\right)-t_{s} \xi\right]
$$

for the approximate PDF of $y$, where the subscript "PS" stands for PDD-SPA and $t_{s}$ is the saddlepoint that is obtained from solving

$$
\tilde{K}_{y, Q, S, m}^{\prime}\left(t_{s} ; \mathbf{d}\right)=\xi
$$

with $\tilde{K}_{y, Q, S, m}^{\prime}(t ; \mathbf{d}):=d \tilde{K}_{y, Q, S, m}(t ; \mathbf{d}) / d t$ and $\tilde{K}_{y, Q, S, m}^{\prime \prime}(t ; \mathbf{d}):=d^{2} \tilde{K}_{y, Q, S, m}(t ; \mathbf{d}) / d t^{2} \operatorname{defining}$ the first- and second-order derivatives, respectively, of the approximate CGF of $y$ with respect to $t$. Furthermore, based on a related work of Lugannani and Rice [19], the approximate CDF of $y$ becomes

$$
\begin{gathered}
\tilde{F}_{y, P S}(\xi ; \mathbf{d})=\Phi(w)+\phi(w)\left(\frac{1}{w}-\frac{1}{v}\right), \\
w=\operatorname{sgn}\left(t_{s}\right)\left\{2\left[t_{s} \xi-\tilde{K}_{y, Q, S, m}\left(t_{s} ; \mathbf{d}\right)\right]\right\}^{\frac{1}{2}}, v=t_{s}\left[\tilde{K}_{y, Q, S, m}^{\prime \prime}\left(t_{s} ; \mathbf{d}\right)\right]^{\frac{1}{2}},
\end{gathered}
$$

where $\Phi(\cdot)$ and $\phi(\cdot)$ are the CDF and PDF, respectively, of the standard Gaussian variable and $\operatorname{sgn}\left(t_{s}\right)=+1,-1$, or 0 , depending on whether $t_{s}$ is positive, negative, or zero. According to Equation 31, the CDF of $y$ at a point $\xi$ is obtained using solely the corresponding saddlepoint $t_{s}$, that is, without the need to integrate Equation 29 from $-\infty$ to $\xi$. 
Finally, using Lugannani and Rice's formula, the PDD-SPA estimate $\tilde{P}_{F, P S}(\mathbf{d})$ of the component failure probability $P_{F}(\mathbf{d}):=P[y(\mathbf{X})<0]$ is obtained as

$$
\tilde{P}_{F, P S}(\mathbf{d})=\tilde{F}_{y, P S}(0 ; \mathbf{d}),
$$

the PDD-SPA generated CDF of $y$ at $\xi=0$. It is important to recognize that no similar SPA-based formulae are available for the joint PDF or joint CDF of dependent stochastic responses. Therefore, the PDD-SPA method in the current form cannot be applied to general system reliability analysis.

The PDD-SPA method contains several truncation parameters that should be carefully selected. For instance, if $Q$ is too small, then the truncated CGF from Equation 28 may spoil the method, regardless of how large are $S$ and $m$ chosen in the PDD approximation. On the other hand, if $Q$ is overly large, then many higher-order moments involved may not be accurately calculated by the PDD approximation. More significantly, a finite-order truncation of CGF may cause loss of convexity of the actual CGF, meaning that the one-to-one relationship between $\xi$ and $t_{s}$ in Equation 30 is not ensured for every threshold $\xi$. Furthermore, the important property $\tilde{K}_{y, Q, S, m}^{\prime \prime}\left(t_{s} ; \mathbf{d}\right)>0$ may not be maintained. To resolve this quandary, Yuen et al. [20] presented for $Q=4$ several distinct cases of the cumulants, describing the interval $\left(t_{l}, t_{u}\right)$, where $-\infty \leq t_{l} \leq 0$ and $0 \leq t_{u} \leq \infty$, such that $t_{l} \leq t_{s} \leq t_{u}$ and $\tilde{K}_{y, Q, S, m}^{\prime \prime}\left(t_{s} ; \mathbf{d}\right)>0$, ruling out any complex values of the square root in Equation 29 or 31 Table 1 summarizes these cases, which were employed in the PDD-SPA method described in this paper. If the specified threshold $\xi \in\left(\tilde{K}^{\prime}{ }_{y, Q, S, m}\left(t_{l} ; \mathbf{d}\right), \tilde{K}^{\prime}{ }_{y, Q, S, m}\left(t_{u} ; \mathbf{d}\right)\right)$, then the saddlepoint $t_{s}$ is uniquely determined from Equation 30 , leading to the CDF or reliability in Equation 31 or 32. Otherwise, the PDD-SPA method will fail to provide a solution. It is important to note that developing similar cases for $Q>4$, assuring a unique solution of the saddlepoint, is not trivial, and was not considered in this work.

Table I. Intervals of the saddlepoint for $Q=4^{(\text {a) }}$

\begin{tabular}{|c|c|c|c|}
\hline Case & Condition & $t_{l}$ & $t_{u}$ \\
\hline 1 & $\tilde{\kappa}_{S, m}^{(4)}>0, \Delta>0, \tilde{\kappa}_{S, m}^{(3)}>0$ & $\frac{-\tilde{\kappa}_{S, m}^{(3)}+\sqrt{\Delta}}{\tilde{\kappa}_{S, m}^{(4)}}$ & $+\infty$ \\
\hline 2 & $\tilde{\kappa}_{S, m}^{(4)}>0, \Delta>0, \tilde{\kappa}_{S, m}^{(3)}<0$ & $-\infty$ & $\frac{-\tilde{\kappa}_{S, m}^{(3)}-\sqrt{\Delta}}{\tilde{\kappa}_{S, m}^{(4)}}$ \\
\hline 3 & $\tilde{\kappa}_{S, m}^{(4)}>0, \Delta=0$ & $-\infty^{(\mathrm{b})}$ & $+\infty(\mathrm{b})$ \\
\hline 4 & $\tilde{\kappa}_{S, m}^{(4)}>0, \Delta<0$ & $-\infty$ & $+\infty$ \\
\hline 5 & $\tilde{\kappa}_{S, m}^{(4)}=0, \tilde{\kappa}_{S, m}^{(3)}>0$ & $-\frac{\tilde{\kappa}_{S, m}^{(2)}}{\tilde{\kappa}_{S, m}^{(3)}}$ & $+\infty$ \\
\hline 6 & $\tilde{\kappa}_{S, m}^{(4)}=0, \tilde{\kappa}_{S, m}^{(3)}=0$ & $-\infty$ & $\begin{array}{r}+\infty \\
\tilde{r}_{(2)}(2)\end{array}$ \\
\hline 7 & $\tilde{\kappa}_{S, m}^{(4)}=0, \tilde{\kappa}_{S, m}^{(3)}<0$ & $-\infty$ & \\
\hline 8 & $\tilde{\kappa}_{S, m}^{(4)}<0$ & $\frac{-\tilde{\kappa}_{S, m}^{(3)}+\sqrt{\Delta}}{\tilde{\kappa}_{S, m}^{(4)}}$ & $\frac{-\tilde{\kappa}_{S, m}^{(3)}-\sqrt{\Delta}}{\tilde{\kappa}_{S, m}^{(4)}}$ \\
\hline \multicolumn{4}{|c|}{$\begin{array}{l}\text { (a) For } \tilde{K}_{y, 4, S, m}(t ; \mathbf{d})=\tilde{\kappa}_{S, m}^{(1)}(\mathbf{d}) t+\frac{1}{2 !} \tilde{\kappa}_{S, m}^{(2)}(\mathbf{d}) t^{2}+\frac{1}{3 !} \tilde{\kappa}_{S, m}^{(3)}(\mathbf{d}) t^{3}+\frac{1}{4 !} \tilde{\kappa}_{S, m}^{(4)}(\mathbf{d}) t^{4} \\
\text { the discriminant of } \tilde{K}_{y, 4, S, m}^{\prime}(t ; \mathbf{d}) \text { is } \Delta:=\tilde{\kappa}_{S, m}^{(3)^{2}}-2 \tilde{\kappa}_{S, m}^{(2)} \tilde{\kappa}_{S, m}^{(4)} \text {. } \\
\text { (b) The point }-\tilde{\kappa}_{S, m}^{(3)} /\left(2 \tilde{\kappa}_{S, m}^{2)}\right) \text { should not be an element of }\left(t_{l}, t_{u}\right) \text {, i.e., }\left(t_{l}, t_{u}\right)= \\
(-\infty, \infty) \backslash\left\{-\tilde{\kappa}_{S, m}^{(3)} /\left(2 \tilde{\kappa}_{S, m}^{(2)}\right)\right\} \text {. }\end{array}$} \\
\hline
\end{tabular}

2.3.2. The PDD-MCS Method Depending on component or system reliability analysis, let $\quad \tilde{\Omega}_{F, S, m}:=\left\{\mathbf{x}: \tilde{y}_{S, m}(\mathbf{x})<0\right\} \quad$ or $\quad \tilde{\Omega}_{F, S, m}:=\left\{\mathbf{x}: \cup_{i} \tilde{y}_{i, S, m}(\mathbf{x})<0\right\} \quad$ or $\quad \tilde{\Omega}_{F, S, m}:=\{\mathbf{x}:$ 
$\left.\cap_{i} \tilde{y}_{i, S, m}(\mathbf{x})<0\right\}$ be an approximate failure set as a result of $S$-variate, $m$ th-order PDD approximations $\tilde{y}_{S, m}(\mathbf{X})$ of $y(\mathbf{X})$ or $\tilde{y}_{i, S, m}(\mathbf{X})$ of $y_{i}(\mathbf{X})$. Then the PDD-MCS estimate of the failure probability $P_{F}(\mathbf{d})$ is

$$
\tilde{P}_{F, P M}(\mathbf{d})=\mathbb{E}_{\mathbf{d}}\left[I_{\tilde{\Omega}_{F, S, m}}(\mathbf{X})\right]=\lim _{L \rightarrow \infty} \frac{1}{L} \sum_{l=1}^{L} I_{\tilde{\Omega}_{F, S, m}}\left(\mathbf{x}^{(l)}\right),
$$

where the subscript "PM" stands for PDD-MCS, $L$ is the sample size, $\mathbf{x}^{(l)}$ is the $l$ th realization of $\mathbf{X}$, and $I_{\tilde{\Omega}_{F, S, m}}(\mathbf{x})$ is another indicator function, which is equal to one when $\mathbf{x} \in \tilde{\Omega}_{F, S, m}$ and zero otherwise.

Note that the simulation of the PDD approximation in Equation 33 should not be confused with crude MCS commonly used for producing benchmark results. The crude MCS, which requires numerical calculations of $y\left(\mathbf{x}^{(l)}\right)$ or $y_{i}\left(\mathbf{x}^{(l)}\right)$ for input samples $\mathbf{x}^{(l)}, l=1, \cdots, L$, can be expensive or even prohibitive, particularly when the sample size $L$ needs to be very large for estimating small failure probabilities. In contrast, the MCS embedded in PDD requires evaluations of simple analytical functions that stem from an $S$-variate, $m$ th-order approximation $\tilde{y}_{S, m}\left(\mathbf{x}^{(l)}\right)$ or $\tilde{y}_{i, S, m}\left(\mathbf{x}^{(l)}\right)$. Therefore, an arbitrarily large sample size can be accommodated in the PDD-MCS method. In which case, the PDD-MCS method also furnishes the approximate CDF $\tilde{F}_{y, P M}(\xi ; \mathbf{d}):=$ $P_{\mathbf{d}}\left[\tilde{y}_{S, m}(\mathbf{X}) \leq \xi\right]$ of $y(\mathbf{X})$ or even joint CDF of dependent stochastic responses, if desired.

Although the PDD-SPA and PDD-MCS methods are both rooted in the same PDD approximation, the former requires additional layers of approximations to calculate the CGF and saddlepoint. Therefore, the PDD-SPA method, when it works, is expected to be less accurate than the PDDMCS method at comparable computational efforts. However, the PDD-SPA method facilitates an analytical means to estimate the probability distribution and reliability - a convenient process not supported by the PDD-MCS method. The respective properties of both methods extend to sensitivity analysis, presented in the following two sections.

\section{DESIGN SENSITIVITY ANALYSIS OF MOMENTS}

When solving RDO problems using gradient-based optimization algorithms, at least first-order derivatives of the first and second moments of a stochastic response with respect to each design variable are required. In this section, a new method, developed by blending PDD with score functions, for design sensitivity analysis of moments of an arbitrary order, is presented.

\subsection{Score Functions}

Suppose that the first-order derivative of a moment $m^{(r)}(\mathbf{d})$, where $r \in \mathbb{N}$, of a generic stochastic response $y(\mathbf{X})$ with respect to a design variable $d_{k}, 1 \leq k \leq M$, is sought. Taking partial derivative of the moment with respect to $d_{k}$ and then applying the Lebesgue dominated convergence theorem [21], which permits the differential and integral operators to be interchanged, yields the sensitivity

$$
\begin{aligned}
\frac{\partial m^{(r)}(\mathbf{d})}{\partial d_{k}} & :=\frac{\partial \mathbb{E}_{\mathbf{d}}\left[y^{r}(\mathbf{X})\right]}{\partial d_{k}} \\
& =\frac{\partial}{\partial d_{k}} \int_{\mathbb{R}^{N}} y^{r}(\mathbf{x}) f_{\mathbf{X}}(\mathbf{x} ; \mathbf{d}) d \mathbf{x} \\
& =\int_{\mathbb{R}^{N}} y^{r}(\mathbf{x}) \frac{\partial \ln f_{\mathbf{X}}(\mathbf{x} ; \mathbf{d})}{\partial d_{k}} f_{\mathbf{X}}(\mathbf{x} ; \mathbf{d}) d \mathbf{x} \\
& =: \mathbb{E}_{\mathbf{d}}\left[y^{r}(\mathbf{X}) s_{d_{k}}^{(1)}(\mathbf{X} ; \mathbf{d})\right]
\end{aligned}
$$

provided that $f_{\mathbf{X}}(\mathbf{x} ; \mathbf{d})>0$ and the derivative $\partial \ln f_{\mathbf{X}}(\mathbf{x} ; \mathbf{d}) / \partial d_{k}$ exists. In last line of Equation 34 , $s_{d_{k}}^{(1)}(\mathbf{X} ; \mathbf{d}):=\partial \ln f_{\mathbf{X}}(\mathbf{X} ; \mathbf{d}) / \partial d_{k}$ is known as the first-order score function for the design variable $d_{k}$ [3, 4]. In general, the sensitivities are not available analytically since the moments are not either. Nonetheless, the moments and their sensitivities have both been formulated as expectations 
of stochastic quantities with respect to the same probability measure, facilitating their concurrent evaluations in a single stochastic simulation or analysis.

Remark 5. The evaluation of score functions, $s_{d_{k}}^{(1)}(\mathbf{X} ; \mathbf{d}), k=1, \cdots, M$, requires differentiating only the PDF of $\mathbf{X}$. Therefore, the resulting score functions can be determined easily and, in many cases, analytically - for instance, when $\mathbf{X}$ follows classical probability distributions [4]. If the density function of $\mathbf{X}$ is arbitrarily prescribed, the score functions can be calculated numerically, yet inexpensively, since no evaluation of the performance function is involved.

When $\mathbf{X}$ comprises independent variables, as assumed here, $\ln f_{\mathbf{X}}(\mathbf{X} ; \mathbf{d})=\sum_{i=1}^{i=N} \ln f_{X_{i}}\left(x_{i} ; \mathbf{d}\right)$ is a sum of $N$ univariate log-density (marginal) functions of random variables. Hence, in general, the score function for the $k$ th design variable, expressed by

$$
s_{d_{k}}^{(1)}(\mathbf{X} ; \mathbf{d})=\sum_{i=1}^{N} \frac{\partial \ln f_{X_{i}}\left(X_{i} ; \mathbf{d}\right)}{\partial d_{k}}=\sum_{i=1}^{N} s_{k i}\left(X_{i} ; \mathbf{d}\right),
$$

is also a sum of univariate functions $s_{k i}\left(X_{i} ; \mathbf{d}\right):=\partial \ln f_{X_{i}}\left(X_{i} ; \mathbf{d}\right) / \partial d_{k}, i=1, \cdots, N$, which are the derivatives of log-density (marginal) functions. If $d_{k}$ is a distribution parameter of a single random variable $X_{i_{k}}$, then the score function reduces to $s_{d_{k}}^{(1)}(\mathbf{X} ; \mathbf{d})=\partial \ln f_{X_{i_{k}}}\left(X_{i_{k}} ; \mathbf{d}\right) / \partial d_{k}=$ : $s_{k i_{k}}\left(X_{i_{k}} ; \mathbf{d}\right)$, the derivative of the log-density (marginal) function of $X_{i_{k}}$, which remains a univariate function. Nonetheless, combining Equations 34 and 35, the sensitivity is obtained from

$$
\frac{\partial m^{(r)}(\mathbf{d})}{\partial d_{k}}=\sum_{i=1}^{N} \mathbb{E}_{\mathbf{d}}\left[y^{r}(\mathbf{X}) s_{k i}\left(X_{i} ; \mathbf{d}\right)\right],
$$

the sum of expectations of products comprising stochastic response and log-density derivative functions with respect to the probability measure $P_{\mathbf{d}}, \mathbf{d} \in \mathcal{D}$.

\subsection{Sensitivities of First- and Second-Order Moments}

For independent coordinates of $\mathbf{X}$, consider the Fourier-polynomial expansion of the $k$ th log-density derivative function

$$
s_{k i}\left(X_{i} ; \mathbf{d}\right)=s_{k i, \emptyset}(\mathbf{d})+\sum_{j=1}^{\infty} D_{k, i j}(\mathbf{d}) \psi_{i j}\left(X_{i} ; \mathbf{d}\right),
$$

consisting of its own expansion coefficients

$$
s_{k i, \emptyset}(\mathbf{d}):=\int_{\mathbb{R}} s_{k i}\left(x_{i} ; \mathbf{d}\right) f_{X_{i}}\left(x_{i} ; \mathbf{d}\right) d x_{i}
$$

and

$$
D_{k, i j}(\mathbf{d}):=\int_{\mathbb{R}} s_{k i}\left(x_{i} ; \mathbf{d}\right) \psi_{i j}\left(x_{i} ; \mathbf{d}\right) f_{X_{i}}\left(x_{i} ; \mathbf{d}\right) d x_{i} .
$$

The expansion is valid if $s_{k i}$ is square integrable with respect to the probability measure of $X_{i}$. When blended with the PDD approximation, the score function leads to analytical or closed-form expressions of the exact or approximate sensitivities as follows.

3.2.1. Exact Sensitivities Employing Equations 11 and 37, the product appearing on the right side of Equation 36 expands to

$$
\begin{aligned}
y^{r}(\mathbf{X}) s_{k i}\left(X_{i} ; \mathbf{d}\right)= & \left(y_{\emptyset}(\mathbf{d})+\sum_{\emptyset \neq u \subseteq\{1, \cdots, N\}} \sum_{\substack{\mathbf{j}_{|u|} \in \mathbb{N}_{0}^{|u|} \\
j_{1}, \cdots, j_{|u|} \neq 0}} C_{u \mathbf{j}_{|u|}}(\mathbf{d}) \psi_{u \mathbf{j}_{|u|}}\left(\mathbf{X}_{u} ; \mathbf{d}\right)\right)^{r} \times \\
& \left(s_{k i, \emptyset}(\mathbf{d})+\sum_{j=1}^{\infty} D_{k, i j}(\mathbf{d}) \psi_{i j}\left(X_{i} ; \mathbf{d}\right)\right),
\end{aligned}
$$


encountering the same orthonormal polynomial bases that are consistent with the probability measure $f_{\mathbf{X}}(\mathbf{x} ; \mathbf{d}) d \mathbf{x}$. The expectations of Equation 40 for $r=1$ and 2, aided by Propositions 2 and 3 , lead Equation 36 to

$$
\frac{\partial m^{(1)}(\mathbf{d})}{\partial d_{k}}=\sum_{i=1}^{N}\left[y_{\emptyset}(\mathbf{d}) s_{k i, \emptyset}(\mathbf{d})+\sum_{j=1}^{\infty} C_{i j}(\mathbf{d}) D_{k, i j}(\mathbf{d})\right]
$$

and

$$
\frac{\partial m^{(2)}(\mathbf{d})}{\partial d_{k}}=\sum_{i=1}^{N}\left[m^{(2)}(\mathbf{d}) s_{k i, \emptyset}(\mathbf{d})+2 y_{\emptyset}(\mathbf{d}) \sum_{j=1}^{\infty} C_{i j}(\mathbf{d}) D_{k, i j}(\mathbf{d})+T_{k i}\right],
$$

representing closed-form expressions of the sensitivities in terms of the PDD or Fourier-polynomial expansion coefficients of the response or log-density derivative functions. The last term on the right side of Equation 42 is

$$
\begin{aligned}
T_{k i}= & \sum_{i_{1}=1}^{N} \sum_{i_{2}=1}^{N} \sum_{j_{1}=1}^{\infty} \sum_{j_{2}=1}^{\infty} \sum_{j_{3}=1}^{\infty} C_{i_{1} j_{1}}(\mathbf{d}) C_{i_{2} j_{2}}(\mathbf{d}) D_{k, i j_{3}}(\mathbf{d}) \times \\
& \mathbb{E}_{\mathbf{d}}\left[\psi_{i_{1} j_{1}}\left(X_{i_{1}} ; \mathbf{d}\right) \psi_{i_{2} j_{2}}\left(X_{i_{2}} ; \mathbf{d}\right) \psi_{i_{3} j_{3}}\left(X_{i} ; \mathbf{d}\right)\right],
\end{aligned}
$$

which requires expectations of various products of three random orthonormal polynomials and is further discussed in Subsection 3.2.4. Note that these sensitivity equations are exact because PDD and Fourier-polynomial expansions are exact representations of square-integrable functions.

3.2.2. Approximate Sensitivities When $y(\mathbf{X})$ and $s_{k i}\left(X_{i} ; \mathbf{d}\right)$ are replaced by their $S$-variate, $m$ thorder PDD and $m^{\prime}$ th-order Fourier-polynomial approximations, respectively, the resultant sensitivity equations, expressed by

$$
\frac{\partial \tilde{m}_{S, m}^{(1)}(\mathbf{d})}{\partial d_{k}}:=\frac{\partial \mathbb{E}_{\mathbf{d}}\left[\tilde{y}_{S, m}(\mathbf{X})\right]}{\partial d_{k}}=\sum_{i=1}^{N}\left[y_{\emptyset}(\mathbf{d}) s_{k i, \emptyset}(\mathbf{d})+\sum_{j=1}^{m_{\min }} C_{i j}(\mathbf{d}) D_{k, i j}(\mathbf{d})\right]
$$

and

$\frac{\partial \tilde{m}_{S, m}^{(2)}(\mathbf{d})}{\partial d_{k}}:=\frac{\partial \mathbb{E}_{\mathbf{d}}\left[\tilde{y}_{S, m}^{2}(\mathbf{X})\right]}{\partial d_{k}}=\sum_{i=1}^{N}\left[\tilde{m}_{S, m}^{(2)}(\mathbf{d}) s_{k i, \emptyset}(\mathbf{d})+2 y_{\emptyset}(\mathbf{d}) \sum_{j=1}^{m_{\min }} C_{i j}(\mathbf{d}) D_{k, i j}(\mathbf{d})+\tilde{T}_{k i, m, m^{\prime}}\right]$,

where $m_{\min }:=\min \left(m, m^{\prime}\right)$ and

$$
\begin{aligned}
\tilde{T}_{k i, m, m^{\prime}}= & \sum_{i_{1}=1}^{N} \sum_{i_{2}=1}^{N} \sum_{j_{1}=1}^{m} \sum_{j_{2}=1}^{m} \sum_{j_{3}=1}^{m^{\prime}} C_{i_{1} j_{1}}(\mathbf{d}) C_{i_{2} j_{2}}(\mathbf{d}) D_{k, i j_{3}}(\mathbf{d}) \times \\
& \mathbb{E}_{\mathbf{d}}\left[\psi_{i_{1} j_{1}}\left(X_{i_{1}} ; \mathbf{d}\right) \psi_{i_{2} j_{2}}\left(X_{i_{2}} ; \mathbf{d}\right) \psi_{i j_{3}}\left(X_{i} ; \mathbf{d}\right)\right],
\end{aligned}
$$

become approximate, relying on the truncation parameters $S, m$, and $m^{\prime}$ in general. At appropriate limits, the approximate sensitivities of the moments converge to exactness as described by Proposition 6 .

Proposition 6. Let $\tilde{y}_{S, m}(\mathbf{X})$ be an $S$-variate, mth-order PDD approximation of a square-integrable function $y(\mathbf{X})$, where $\mathbf{X}=\left(X_{1}, \cdots, X_{N}\right) \in \mathbb{R}^{N}$ comprises independent random variables with marginal probability distributions $f_{X_{i}}\left(x_{i} ; \mathbf{d}\right), i=1, \cdots, N$, and $\mathbf{d}=\left(d_{1}, \cdots, d_{M}\right) \in \mathcal{D}$ is a design vector with non-empty closed set $\mathcal{D} \subseteq \mathbb{R}^{M}$. Given the distribution parameter $d_{k}$, let the kth logdensity derivative function $s_{k i}\left(X_{i} ; \mathbf{d}\right)$ of the ith random variable $X_{i}$ be square integrable. Then for $k=1, \cdots M$,

$$
\lim _{S \rightarrow N, m, m^{\prime} \rightarrow \infty} \frac{\partial \tilde{m}_{S, m}^{(1)}(\mathbf{d})}{\partial d_{k}}=\frac{\partial m^{(1)}(\mathbf{d})}{\partial d_{k}}
$$


and

$$
\lim _{S \rightarrow N, m, m^{\prime} \rightarrow \infty} \frac{\partial \tilde{m}_{S, m}^{(2)}(\mathbf{d})}{\partial d_{k}}=\frac{\partial m^{(2)}(\mathbf{d})}{\partial d_{k}} .
$$

Proof

Taking the limits $S \rightarrow N, m \rightarrow \infty$, and $m^{\prime} \rightarrow \infty$ on Equations 44 and 45 and recognizing $\tilde{m}_{S, m}^{(2)}(\mathbf{d}) \rightarrow m^{(2)}(\mathbf{d})$ and $\tilde{T}_{k i, m, m^{\prime}} \rightarrow T_{k i}$,

$$
\begin{aligned}
\lim _{S \rightarrow N, m, m^{\prime} \rightarrow \infty} \frac{\partial \tilde{m}_{S, m}^{(1)}(\mathbf{d})}{\partial d_{k}} & =\lim _{S \rightarrow N, m, m^{\prime} \rightarrow \infty} \sum_{i=1}^{N}\left[y_{\emptyset}(\mathbf{d}) s_{k i, \emptyset}(\mathbf{d})+\sum_{j=1}^{m_{\min }} C_{i j}(\mathbf{d}) D_{k, i j}(\mathbf{d})\right] \\
& =\sum_{i=1}^{N}\left[y_{\emptyset}(\mathbf{d}) s_{k i, \emptyset}(\mathbf{d})+\sum_{j=1}^{\infty} C_{i j}(\mathbf{d}) D_{k, i j}(\mathbf{d})\right] \\
& =\frac{\partial m^{(1)}(\mathbf{d})}{\partial d_{k}}
\end{aligned}
$$

and

$$
\begin{aligned}
& \lim _{s \rightarrow N, m_{m} \rightarrow \infty} \frac{\partial \tilde{m}_{S, m}^{(2)}(\mathbf{d})}{\partial d_{k}} \\
= & \lim _{S \rightarrow N, m^{\prime} \rightarrow \infty} \sum_{i=1}^{N}\left[\tilde{m}_{S, m}^{(2)^{2}}(\mathbf{d}) s_{k i, \emptyset}(\mathbf{d})+2 y_{\emptyset}(\mathbf{d}) \sum_{j=1}^{m_{\min }} C_{i j}(\mathbf{d}) D_{k, i j}(\mathbf{d})+\tilde{T}_{k i, m, m^{\prime}}\right] \\
= & \sum_{i=1}^{N}\left[m^{(2)^{2}}(\mathbf{d}) s_{k i, \emptyset}(\mathbf{d})+2 y_{\emptyset}(\mathbf{d}) \sum_{j=1}^{\infty} C_{i j}(\mathbf{d}) D_{k, i j}(\mathbf{d})+T_{k i}\right] \\
= & \frac{\partial m^{(2)}(\mathbf{d})}{\partial d_{k}},
\end{aligned}
$$

where the last lines follow from Equations 41 and 42

Of the two sensitivities, $\partial \tilde{m}_{S, m}^{(1)}(\mathbf{d}) / \partial d_{k}$ does not depend on $S$, meaning that both the univariate $(S=1)$ and bivariate $(S=2)$ approximations, given the same $m_{\min }<\infty$, form the same result, as displayed in Equation 44 However, the sensitivity equations of $\partial \tilde{m}_{S, m}^{(2)}(\mathbf{d}) / \partial d_{k}$ for the univariate and bivariate approximations vary with respect to $S, m$, and $m^{\prime}$. For instance, the univariate approximation results in

$$
\frac{\partial \tilde{m}_{1, m}^{(2)}(\mathbf{d})}{\partial d_{k}}=\sum_{i=1}^{N}\left[\tilde{m}_{1, m}^{(2)}(\mathbf{d}) s_{k i, \emptyset}(\mathbf{d})+2 y_{\emptyset}(\mathbf{d}) \sum_{j=1}^{m_{\min }} C_{i j}(\mathbf{d}) D_{k, i j}(\mathbf{d})+\tilde{T}_{k i, m, m^{\prime}}\right],
$$

whereas the bivariate approximation yields

$$
\frac{\partial \tilde{m}_{2, m}^{(2)}(\mathbf{d})}{\partial d_{k}}=\sum_{i=1}^{N}\left[\tilde{m}_{2, m}^{(2)}(\mathbf{d}) s_{k i, \emptyset}(\mathbf{d})+2 y_{\emptyset}(\mathbf{d}) \sum_{j=1}^{m_{\min }} C_{i j}(\mathbf{d}) D_{k, i j}(\mathbf{d})+\tilde{T}_{k i, m, m^{\prime}}\right] .
$$

Analogous to the moments, the univariate and bivariate approximations of the sensitivities of the moments involve only univariate and at most bivariate expansion coefficients of $y$, respectively. Since the expansion coefficients of log-density derivative functions do not involve the response function, no additional cost is incurred from response analysis. In other words, the effort required to obtain the statistical moments of a response also furnishes the sensitivities of moments, a highly desirable trait for efficiently solving RDO problems. 
Remark 7. Since the derivatives of log-density functions are univariate functions, their expansion coefficients require only univariate integration for their evaluations. When $X_{i}$ follows classical distributions - for instance, the Gaussian distribution - then the coefficients can be calculated exactly or analytically. Otherwise, numerical quadrature is required. Nonetheless, there is no need to employ dimension-reduction integration for calculating the expansion coefficients of the derivatives of log-density functions.

3.2.3. Special Cases There exist two special cases when the preceding expressions of the sensitivities of moments simplify slightly. They are contingent on how a distribution parameter affects the probability distributions of random variables.

First, when $\mathbf{X}$ comprises independent variables such that $d_{k}$ is a distribution parameter of a single random variable, say, $X_{i_{k}}, 1 \leq i_{k} \leq N$, then $s_{k i_{k}}\left(X_{i_{k}} ; \mathbf{d}\right)-$ the $k$ th log-density derivative function of $X_{i_{k}}$ - is the only relevant function of interest. Consequently, the expansion coefficients $s_{k i, \emptyset}(\mathbf{d})=s_{k i_{k}, \emptyset}(\mathbf{d})$ (say) and $D_{k, i j}(\mathbf{d})=D_{k, i_{k} j}(\mathbf{d})$ (say), if $i=i_{k}$ and zero otherwise. Moreover, the outer sums of Equations 44 and 45 vanish, yielding

$$
\frac{\partial \tilde{m}_{S, m}^{(1)}(\mathbf{d})}{\partial d_{k}}=y_{\emptyset}(\mathbf{d}) s_{k i_{k}, \emptyset}(\mathbf{d})+\sum_{j=1}^{m_{\min }} C_{i_{k} j}(\mathbf{d}) D_{k, i_{k} j}(\mathbf{d})
$$

and

$$
\frac{\partial \tilde{m}_{S, m}^{(2)}(\mathbf{d})}{\partial d_{k}}=\tilde{m}_{S, m}^{(2)}(\mathbf{d}) s_{k i_{k}, \emptyset}(\mathbf{d})+2 y_{\emptyset}(\mathbf{d}) \sum_{j=1}^{m_{\min }} C_{i_{k} j}(\mathbf{d}) D_{k, i_{k} j}(\mathbf{d})+\tilde{T}_{k i_{k}, m, m^{\prime}} .
$$

Second, when $\mathbf{X}$ consists of independent and identical variables, then $s_{k i}\left(X_{i} ; \mathbf{d}\right)=s_{k}\left(X_{i} ; \mathbf{d}\right)$ (say), that is, the $k$ th $\log$-density derivative functions of all random variables are alike. Accordingly, the expansion coefficients $s_{k i, \emptyset}(\mathbf{d})=s_{k, \emptyset}(\mathbf{d})$ (say) and $D_{k, i j}(\mathbf{d})=D_{k, j}(\mathbf{d})$ (say) for all $i=$ $1, \cdots, N$, producing

$$
\frac{\partial \tilde{m}_{S, m}^{(1)}(\mathbf{d})}{\partial d_{k}}=\sum_{i=1}^{N}\left[y_{\emptyset}(\mathbf{d}) s_{k, \emptyset}(\mathbf{d})+\sum_{j=1}^{m_{\min }} C_{i j}(\mathbf{d}) D_{k, j}(\mathbf{d})\right]
$$

and

$$
\frac{\partial \tilde{m}_{S, m}^{(2)}(\mathbf{d})}{\partial d_{k}}=\sum_{i=1}^{N}\left[\tilde{m}_{S, m}^{(2)}(\mathbf{d}) s_{k, \emptyset}(\mathbf{d})+2 y_{\emptyset}(\mathbf{d}) \sum_{j=1}^{m_{\min }} C_{i j}(\mathbf{d}) D_{k, j}(\mathbf{d})+\tilde{T}_{k i, m, m^{\prime}}\right] .
$$

It is important to clarify that the first special case, that is, Equations 53 and 54 , coincide with those presented in a previous work [22] by the authors. However, the second case, that is, Equations 55 and 56 including the generalized version, that is, Equations 44 and 45 , are new. The results of sensitivity equations from these two special cases will be discussed in the Numerical Examples section.

3.2.4. Evaluation of $\tilde{T}_{k i, m, m^{\prime}}$ The evaluation of $\tilde{T}_{k i, m, m^{\prime}}$ in Equation 46 requires expectations of various products of three random orthonormal polynomials. The expectations vanish when $i_{1} \neq i_{2} \neq i_{3}$, regardless of the probability measures of random variables. For classical polynomials, such as Hermite, Laguerre, and Legendre polynomials, there exist formulae for calculating the expectations when $i_{1}=i_{2}=i_{3}=i$ (say).

When $X_{i}$ follows the standard Gaussian distribution, the expectations are determined from the properties of univariate Hermite polynomials, yielding [23]

$$
\mathbb{E}_{\mathbf{d}}\left[\psi_{i j_{1}}\left(X_{i} ; \mathbf{d}\right) \psi_{i j_{2}}\left(X_{i} ; \mathbf{d}\right) \psi_{i j_{3}}\left(X_{i} ; \mathbf{d}\right)\right]=\frac{\sqrt{j_{1} ! j_{2} ! j_{3} !}}{\left(q-j_{1}\right) !\left(q-j_{2}\right) !\left(q-j_{3}\right) !},
$$

if $q \in \mathbb{N}, 2 q=j_{1}+j_{2}+j_{3}$, and $j_{1}, j_{2}, j_{3} \leq q$, and zero otherwise. When $X_{i}$ follows the exponential distribution with unit mean, the expectations are attained from the properties of univariate Laguerre 
polynomials, producing [24]

$$
\begin{aligned}
& \mathbb{E}_{\mathbf{d}}\left[\psi_{i j_{1}}\left(X_{i} ; \mathbf{d}\right) \psi_{i j_{2}}\left(X_{i} ; \mathbf{d}\right) \psi_{i j_{3}}\left(X_{i} ; \mathbf{d}\right)\right] \\
= & (-1)^{j_{1}+j_{2}+j_{3}} \sum_{v=v_{\min }}^{v_{\max }} \frac{\left(j_{1}+j_{2}-v\right) ! 2^{j_{3}-j_{1}-j_{2}+2 v}}{v !\left(j_{1}-v\right) !\left(j_{2}-v\right) !}\left(\begin{array}{c}
v \\
j_{3}-j_{1}-j_{2}+2 v
\end{array}\right),
\end{aligned}
$$

if $\left|j_{1}-j_{2}\right| \leq j_{3} \leq j_{1}+j_{2}$, and zero otherwise, where $v_{\min }=\frac{1}{2}\left(j_{1}+j_{2}+1-j_{3}\right), \quad v_{\max }=$ $\min \left(j_{1}, j_{2}, j_{1}+j_{2}-j_{3}\right)$. When $X_{i}$ follows the uniform distribution on the interval $[-1,1]$, the expectations are obtained from the properties of univariate Legendre polynomials, forming [24]

$$
\begin{aligned}
& \mathbb{E}_{\mathbf{d}}\left[\psi_{i j_{1}}\left(X_{i} ; \mathbf{d}\right) \psi_{i j_{2}}\left(X_{i} ; \mathbf{d}\right) \psi_{i j_{3}}\left(X_{i} ; \mathbf{d}\right)\right] \\
= & \frac{1}{2} \sqrt{2\left(2 j_{1}+1\right)\left(2 j_{2}+1\right)\left(2 j_{3}+1\right)} \times \\
& \frac{\left(j_{1}+j_{2}-j_{3}-1\right) ! !\left(j_{2}+j_{3}-j_{1}-1\right) ! !\left(j_{1}+j_{2}+j_{3}\right) ! !\left(j_{1}+j_{3}-j_{2}-1\right) ! !}{\left(j_{1}+j_{2}-j_{3}\right) ! !\left(j_{2}+j_{3}-j_{1}\right) ! !\left(j_{1}+j_{2}+j_{3}+1\right) ! !\left(j_{1}+j_{3}-j_{2}\right) ! !}
\end{aligned}
$$

if $q \in \mathbb{N}, 2 q=j_{1}+j_{2}+j_{3}$, and $\left|j_{1}-j_{2}\right| \leq j_{3} \leq j_{1}+j_{2}$, and zero otherwise. The symbol !! in Equation 59 denotes the double factorial. However, deriving a master formula for arbitrary probability distributions of $X_{i}$ is impossible. In which case, the non-trivial solution of the expectation can be obtained by numerical integration of elementary functions.

\subsection{Sensitivities of Higher-Order Moments}

No closed-form or analytical expressions are possible for calculating sensitivities of higher-order $(2<r<\infty)$ moments by the PDD approximation. Two options, consistent with statistical moment analysis in Subsection 2.2, are proposed for sensitivity analysis.

In Option I, the sensitivity is obtained by replacing $y$ by $\tilde{y}_{S, m}$ in Equation 34 and utilizing Equations 20 and 35, resulting in

$$
\begin{aligned}
\frac{\partial \tilde{m}_{S, m}^{(r)}(\mathbf{d})}{\partial d_{k}}= & \int_{\mathbb{R}^{N}} \tilde{y}_{S, m}^{r}(\mathbf{x}) s_{d_{k}}^{(1)}(\mathbf{x} ; \mathbf{d}) f_{\mathbf{X}}(\mathbf{x} ; \mathbf{d}) d \mathbf{x} \\
= & g_{\emptyset}(\mathbf{d}) \sum_{i=1}^{N} \int_{\mathbb{R}} s_{k i}\left(x_{i} ; \mathbf{d}\right) f_{X_{i}}\left(x_{i} ; \mathbf{d}\right) d x_{i}+ \\
& \sum_{i=1}^{N} \sum_{\substack{\emptyset \neq u \subseteq\{1, \cdots, N\}, i \in u \\
1 \leq|u| \leq \min (r S, N)}} \int_{\mathbb{R}^{|u|}} g_{u}\left(\mathbf{x}_{u} ; \mathbf{d}\right) s_{k i}\left(x_{i} ; \mathbf{d}\right) f_{\mathbf{X}_{u}}\left(\mathbf{x}_{u} ; \mathbf{d}\right) d \mathbf{x}_{u}+ \\
& \sum_{i=1}^{N} \sum_{\substack{\emptyset \neq u \subseteq\{1, \cdots, N\}, i \notin u \\
1 \leq|u| \leq \min (r S, N)}} \int_{\mathbb{R}^{|u|}} g_{u}\left(\mathbf{x}_{u} ; \mathbf{d}\right) f_{\mathbf{X}_{u}}\left(\mathbf{x}_{u} ; \mathbf{d}\right) d \mathbf{x}_{u} \times \\
& \int_{\mathbb{R}} s_{k i}\left(x_{i} ; \mathbf{d}\right) f_{X_{i}}\left(x_{i} ; \mathbf{d}\right) d x_{i},
\end{aligned}
$$

which involves at most $\min (r S, N)$-dimensional integrations. Similar to statistical moment analysis, this option becomes impractical when $\min (r S, N)$ is large or numerous $\min (r S, N)$-dimensional integrations are required. 
In contrast, the sensitivity in Option II is attained by replacing $\tilde{y}_{S, m}^{r}$ by $\tilde{z}_{\bar{S}, \bar{m}}$ in the first line of Equation 60, yielding

$$
\begin{aligned}
& \frac{\partial \tilde{m}_{S, m}^{(r)}(\mathbf{d})}{\partial d_{k}} \\
\cong & \int_{\mathbb{R}^{N}} \tilde{z}_{\bar{S}, \bar{m}}(\mathbf{x}) s_{d_{k}}^{(1)}(\mathbf{x} ; \mathbf{d}) f_{\mathbf{X}}(\mathbf{x} ; \mathbf{d}) d \mathbf{x} \\
= & z_{\emptyset}(\mathbf{d}) \sum_{i=1}^{N} \int_{\mathbb{R}} s_{k i}\left(x_{i} ; \mathbf{d}\right) f_{X_{i}}\left(x_{i} ; \mathbf{d}\right) d x_{i}+ \\
& \sum_{i=1}^{N} \sum_{\substack{\emptyset \neq u \subseteq\{1, \cdots, N\} \\
1 \leq|u| \leq \bar{S}, i \in u}} \sum_{\substack{\mathbf{j}_{|u|} \in \mathbb{N}_{0}^{|u|},\left\|\mid \mathbf{j}_{|u|}\right\|_{\infty} \leq \bar{m} \\
j_{1}, \cdots, j_{|u|} \neq 0}} \bar{C}_{u \mathbf{j}_{|u|}}(\mathbf{d}) \int_{\mathbb{R}^{|u|}} \psi_{u \mathbf{j}_{|u|}}\left(\mathbf{x}_{u} ; \mathbf{d}\right) s_{k i}\left(x_{i} ; \mathbf{d}\right) f_{\mathbf{X}_{u}}\left(\mathbf{x}_{u} ; \mathbf{d}\right) d \mathbf{x}_{u}
\end{aligned}
$$

requiring at most $\bar{S}$-dimensional integrations of at most $\bar{m}$ th-order polynomials, where the terms related to $i \notin u$ vanish as per Proposition 2. Therefore, a significant gain in efficiency is possible in Option II for sensitivity analysis as well. The sensitivity equations further simplify for special cases, as explained in Section 3.2. Nonetheless, numerical integrations are necessary for calculating the sensitivities by either option.

\section{DESIGN SENSITIVITY ANALYSIS OF RELIABILITY}

When solving RBDO problems using gradient-based optimization algorithms, at least first-order derivatives of the failure probability with respect to each design variable is required. Two methods for the sensitivity analysis of the failure probability, named the PDD-SPA and PDD-MCS methods, are presented.

\subsection{The PDD-SPA Method}

Suppose that the first-order derivative $\partial \tilde{F}_{y, P S}(\xi ; \mathbf{d}) / \partial d_{k}$ of the $\operatorname{CDF} \tilde{F}_{y, P S}(\xi ; \mathbf{d})$ of $\tilde{y}_{S, m}(\mathbf{X})$, obtained by the PDD-SPA method, with respect to a design variable $d_{k}$, is desired. Applying the chain rule on the derivative of Equation 31 .

$$
\frac{\partial \tilde{F}_{y, P S}(\xi ; \mathbf{d})}{\partial d_{k}}=\sum_{r=1}^{Q}\left(\frac{\partial \tilde{F}_{y, P S}}{\partial w} \frac{\partial w}{\partial \tilde{\kappa}_{S, m}^{(r)}}+\frac{\partial \tilde{F}_{y, P S}}{\partial v} \frac{\partial v}{\partial \tilde{\kappa}_{S, m}^{(r)}}\right) \frac{\partial \tilde{\kappa}_{S, m}^{(r)}}{\partial d_{k}}
$$

is obtained via the partial derivatives

$$
\begin{gathered}
\frac{\partial \tilde{F}_{y, P S}}{\partial w}=\phi(w)\left(\frac{w}{v}-\frac{1}{w^{2}}\right), \frac{\partial \tilde{F}_{y, P S}}{\partial v}=\frac{\phi(w)}{v^{2}}, \\
\frac{\partial \tilde{\kappa}_{S, m}^{(r)}}{\partial d_{k}}= \begin{cases}\frac{\partial \tilde{m}_{S, m}^{(1)}(\mathbf{d})}{\partial d_{k}} & : r=1, \\
\frac{\partial \tilde{m}_{S, m}^{(r)}(\mathbf{d})}{\partial d_{k}}-\sum_{p=1}^{r-1}\left(\begin{array}{l}
r-1 \\
p-1
\end{array}\right)\left(\frac{\partial \tilde{\kappa}_{S, m}^{(r)}}{\partial d_{k}} \tilde{m}_{S, m}^{(r-p)}(\mathbf{d})+\tilde{\kappa}_{S, m}^{(p)} \frac{\partial \tilde{m}_{S, m}^{(r-p)}}{\partial d_{k}}\right) & : 2 \leq r \leq Q,\end{cases}
\end{gathered}
$$

where the derivatives of moments, that is, $\partial \tilde{m}_{S, m}^{(r)} / \partial d_{k}, r=1, \cdots, Q$, required to calculate the derivatives of cumulants, are obtained using score functions, as described in Section 3. The remaining two partial derivatives are expressed by

$$
\frac{\partial w}{\partial \tilde{\kappa}_{S, m}^{(r)}}=\frac{\partial w}{\partial t_{s}} \frac{\partial t_{s}}{\partial \tilde{\kappa}_{S, m}^{(r)}}+\frac{\partial w}{\partial \tilde{K}_{y, Q, S, m}}\left[\frac{\partial \tilde{K}_{y, Q, S, m}}{\partial \tilde{\kappa}_{S, m}^{(r)}}+\frac{\partial \tilde{K}_{y, Q, S, m}}{\partial t_{s}} \frac{\partial t_{s}}{\partial \tilde{\kappa}_{S, m}^{(r)}}\right],
$$


and

$$
\frac{\partial v}{\partial \tilde{\kappa}_{S, m}^{(r)}}=\frac{\partial v}{\partial t_{s}} \frac{\partial t_{s}}{\partial \tilde{\kappa}_{S, m}^{(r)}}+\frac{\partial v}{\partial \tilde{K}_{y, Q, S, m}^{\prime \prime}}\left[\frac{\partial \tilde{K}_{y, Q, S, m}^{\prime \prime}}{\partial \tilde{\kappa}_{S, m}^{(r)}}+\frac{\partial \tilde{K}_{y, Q, S, m}^{\prime \prime}}{\partial t_{s}} \frac{\partial t_{s}}{\partial \tilde{\kappa}_{S, m}^{(r)}}\right],
$$

where

$$
\begin{gathered}
\frac{\partial w}{\partial t_{s}}=\frac{\xi}{w}, \frac{\partial w}{\partial \tilde{K}_{y, Q, S, m}}=-\frac{1}{w}, \frac{\partial \tilde{K}_{y, Q, S, m}}{\partial t_{s}}=\xi, \frac{\partial v}{\partial t_{s}}=\left[\tilde{K}_{y, Q, S, m}^{\prime \prime}\right]^{\frac{1}{2}} \\
\frac{\partial v}{\partial \tilde{K}_{y, Q, S, m}^{\prime \prime}}=\frac{\frac{\partial \tilde{K}_{y, Q, S, m}^{\prime}}{\partial \tilde{\kappa}_{S, m}^{(r)}}}{2 \sqrt{\tilde{K}_{y, Q, S, m}^{\prime \prime}}}, \frac{\partial t_{s}}{\partial \tilde{\kappa}_{S, m}^{(r)}}=-\frac{\partial \tilde{K}_{y, Q, S, m}^{\prime}}{\partial t_{s}}
\end{gathered}
$$

The expressions of the partial derivatives $\partial \tilde{K}_{y, Q, S, m} / \partial \tilde{\kappa}_{S, m}^{(r)}, \partial \tilde{K}_{y, Q, S, m}^{\prime} / \partial \tilde{\kappa}_{S, m}^{(r)}$, and $\partial \tilde{K}_{y, Q, S, m}^{\prime \prime} / \partial \tilde{\kappa}_{S, m}^{(r)}$, not explicitly presented here, can be easily derived from Equation 28 once the cumulants $\tilde{\kappa}_{S, m}^{(r)}, r=1, \cdots, Q$, and the saddlepoint $t_{s}$ are obtained. Similar sensitivity equations were reported by Huang and Zhang [11]. However, Equation 62 is built on the PDD approximation of a stochastic response, as opposed to the RDD approximation used by Huang and Zhang. Furthermore, no transformations of random variables are necessary in the proposed PDD-SPA method.

Henceforth, the first-order derivative of the failure probability estimate by the PDD-SPA method is easily determined from

$$
\frac{\partial \tilde{P}_{F, P S}(\mathbf{d})}{\partial d_{k}}=\frac{\partial \tilde{F}_{y, P S}(0 ; \mathbf{d})}{\partial d_{k}}
$$

the sensitivity of the probability distribution evaluated at $\xi=0$. Algorithm 1 describes the procedure of the PDD-SPA method for calculating the reliability and its design sensitivity of a general stochastic problem.

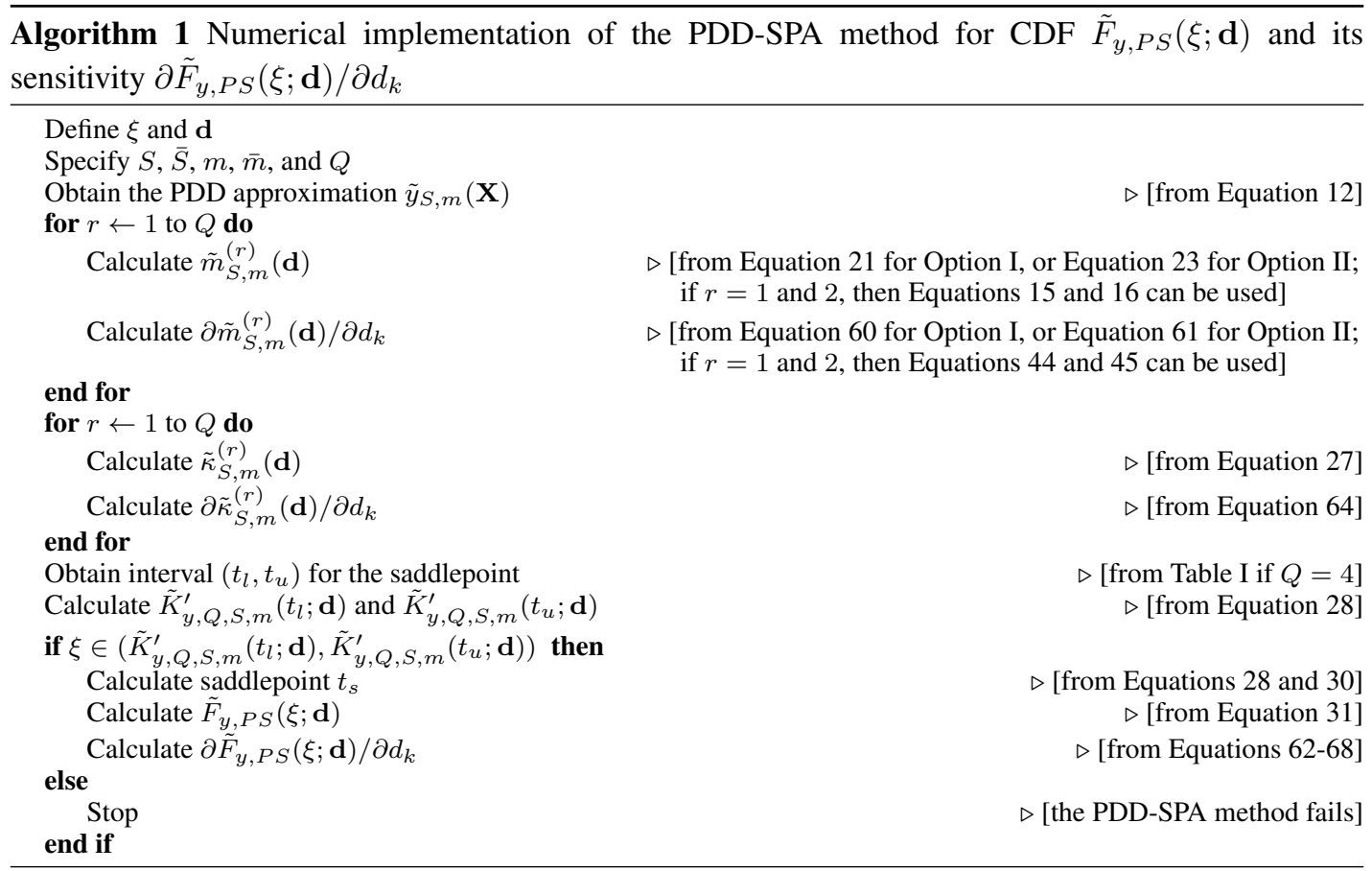




\subsection{The PDD-MCS Method}

Taking a partial derivative of the PDD-MCS estimate of the failure probability in Equation 33 with respect to $d_{k}$ and then following the same arguments in deriving Equation 34 produces

$$
\begin{aligned}
\frac{\partial \tilde{P}_{F, P M}(\mathbf{d})}{\partial d_{k}} & :=\frac{\partial \mathbb{E}_{\mathbf{d}}\left[I_{\tilde{\Omega}_{F, S, m}}(\mathbf{X})\right]}{\partial d_{k}} \\
& =\mathbb{E}_{\mathbf{d}}\left[I_{\tilde{\Omega}_{F, S, m}}(\mathbf{X}) s_{d_{k}}^{(1)}(\mathbf{X} ; \mathbf{d})\right] \\
& =\lim _{L \rightarrow \infty} \frac{1}{L} \sum_{l=1}^{L}\left[I_{\tilde{\Omega}_{F, S, m}}\left(\mathbf{x}^{(l)}\right) s_{d_{k}}^{(1)}\left(\mathbf{x}^{(l)} ; \mathbf{d}\right)\right],
\end{aligned}
$$

where $L$ is the sample size, $\mathbf{x}^{(l)}$ is the $l$ th realization of $\mathbf{X}$, and $I_{\tilde{\Omega}_{F, S, m}}(\mathbf{x})$ is the PDD-generated indicator function, which is equal to one when $\mathbf{x} \in \tilde{\Omega}_{F, S, m}$ and zero otherwise. Again, they are easily and inexpensively determined by sampling analytical functions that describe $\tilde{y}_{S, m}$ and $s_{d_{k}}^{(1)}$. A similar sampling procedure can be employed to calculate the sensitivity of the PDD-MCS generated $\operatorname{CDF} \tilde{F}_{y, P M}(\xi ; \mathbf{d}):=P_{\mathbf{d}}\left[\tilde{y}_{S, m}(\mathbf{X}) \leq \xi\right]$. It is important to note that the effort required to calculate the failure probability or $\mathrm{CDF}$ also delivers their sensitivities, incurring no additional cost. Setting $S=1$ or 2 in Equations 33 and 70 , the univariate or bivariate approximation of the failure probability and its sensitivities are determined.

Remark 8. It is important to recognize that no Fourier-polynomial expansions of the derivatives of log-density functions are required or invoked in the PDD-MCS method for sensitivity analysis of failure probability. This is in contrast to the sensitivity analysis of the first two moments, where such Fourier-polynomial expansions aid in generating analytical expressions of the sensitivities. No analytical expressions are possible in the PDD-MCS method for sensitivity analysis of reliability or probability distribution of a general stochastic response.

Remark 9. The score function method has the nice property that it requires differentiating only the underlying PDF $f_{\mathbf{X}}(\mathbf{x} ; \mathbf{d})$. The resulting score functions can be easily and, in most cases, analytically determined. If the performance function is not differentiable or discontinuous - for example, the indicator function that comes from reliability analysis - the proposed method still allows evaluation of the sensitivity if the density function is differentiable. In reality, the density function is often smoother than the performance function, and therefore the proposed sensitivity methods will be able to calculate sensitivities for a wide variety of complex mechanical systems.

\section{CALCULATION OF EXPANSION COEFFICIENTS}

The determination of PDD expansion coefficients $y_{\emptyset}(\mathbf{d})$ and $C_{u \mathbf{j}_{|u|}}(\mathbf{d})$, where $\emptyset \neq u \subseteq\{1, \cdots, N\}$ and $\mathbf{j}_{|u|} \in \mathbb{N}_{0}^{|u|} \mid ;\left\|\mathbf{j}_{|u|}\right\|_{\infty} \leq m ; j_{1}, \cdots, j_{|u|} \neq 0$, is vitally important for evaluating the statistical moments and probabilistic characteristics, including their design sensitivities, of stochastic responses. The coefficients, defined in Equations 2 and 8 , involve various $N$-dimensional integrals over $\mathbb{R}^{N}$. For large $N$, a full numerical integration employing an $N$-dimensional tensor product of a univariate quadrature formula is computationally prohibitive and is, therefore, ruled out. The authors propose that the dimension-reduction integration scheme, developed by $\mathrm{Xu}$ and Rahman [13], followed by numerical quadrature, be used to estimate the coefficients accurately and efficiently.

\subsection{Dimension-Reduction Integration}

Let $\mathbf{c}=\left(c_{1}, \cdots, c_{N}\right) \in \mathbb{R}^{N}$, which is commonly adopted as the mean of $\mathbf{X}$, be a reference point, and $y\left(\mathbf{x}_{v}, \mathbf{c}_{-v}\right)$ represent an $|v|$-variate $\mathrm{RDD}$ component function of $y(\mathbf{x})$, where $v \subseteq\{1, \cdots, N\}$ [8 14]. Given a positive integer $S \leq R \leq N$, when $y(\mathbf{x})$ in Equations 2 and 8 is replaced with its $R$-variate 
RDD approximation, the coefficients $y_{\emptyset}(\mathbf{d})$ and $C_{u \mathbf{j}_{|u|}}(\mathbf{d})$ are estimated from [13]

$$
y_{\emptyset}(\mathbf{d}) \cong \sum_{i=0}^{R}(-1)^{i}\left(\begin{array}{c}
N-R+i-1 \\
i
\end{array}\right) \sum_{\substack{v \subseteq\{1, \cdots, N\} \\
|v|=R-i}} \int_{\mathbb{R}|v|} y\left(\mathbf{x}_{v}, \mathbf{c}_{-v}\right) f_{\mathbf{X}_{v}}\left(\mathbf{x}_{v} ; \mathbf{d}\right) d \mathbf{x}_{v}
$$

and

$$
C_{u \mathbf{j}_{|u|}}(\mathbf{d}) \cong \sum_{i=0}^{R}(-1)^{i}\left(\begin{array}{c}
N-R+i-1 \\
i
\end{array}\right) \sum_{\substack{v \subseteq\{1, \cdots, N\} \\
|v|=R-i, u \subseteq v}} \int_{\mathbb{R}|v|} y\left(\mathbf{x}_{v}, \mathbf{c}_{-v}\right) \psi_{u \mathbf{j}_{|u|}}\left(\mathbf{x}_{u}\right) f_{\mathbf{X}_{v}}\left(\mathbf{x}_{v} ; \mathbf{d}\right) d \mathbf{x}_{v}
$$

respectively, requiring evaluation of at most $R$-dimensional integrals. The reduced integration facilitates calculation of the coefficients approaching their exact values as $R \rightarrow N$, and is significantly more efficient than performing one $N$-dimensional integration, particularly when $R \ll N$. Hence, the computational effort is significantly lowered using the dimension-reduction integration. For instance, when $R=1$ or 2, Equations 71 and 72 involve one-, or at most, twodimensional integrations, respectively.

For a general function $y$, numerical integrations are still required for performing various $|v|$ dimensional integrals over $\mathbb{R}^{|v|}, 0 \leq|v| \leq R$, in Equations 71 and 72 . When $R>1$, multivariate numerical integrations are conducted by constructing a tensor product of underlying univariate quadrature rules. For a given $v \subseteq\{1, \cdots, N\}, 1<|v| \leq R$, let $v=\left\{i_{1}, \cdots i_{|v|}\right\}$, where $1 \leq$ $i_{1}<\cdots<i_{|v|} \leq N$. Denote by $\left\{x_{i_{p}}^{(1)}, \cdots, x_{i_{p}}^{(n)}\right\} \subset \mathbb{R}$ a set of integration points of $x_{i_{p}}$ and by $\left\{w_{i_{p}}^{(1)}, \cdots, w_{i_{p}}^{(n)}\right\}$ the associated weights generated from a chosen univariate quadrature rule and a positive integer $n \in \mathbb{N}$. Denote by $P^{(n)}=\times_{p=1}^{p=|v|}\left\{x_{i_{p}}^{(1)}, \cdots, x_{i_{p}}^{(n)}\right\}$ a rectangular grid consisting of all integration points generated by the variables indexed by the elements of $v$. Then the coefficients using dimension-reduction integration and numerical quadrature are approximated by

$$
y_{\emptyset}(\mathbf{d}) \cong \sum_{i=0}^{R}(-1)^{i}\left(\begin{array}{c}
N-R+i-1 \\
i
\end{array}\right) \sum_{\substack{v \subseteq\{1, \cdots, N\} \\
|v|=R-i}} \sum_{\mathbf{k}_{|v|} \in P^{(n)}} w^{\left(\mathbf{k}_{|v|}\right)} y\left(\mathbf{x}_{v}^{\left(\mathbf{k}_{|v|}\right)}, \mathbf{c}_{-v}\right)
$$

and

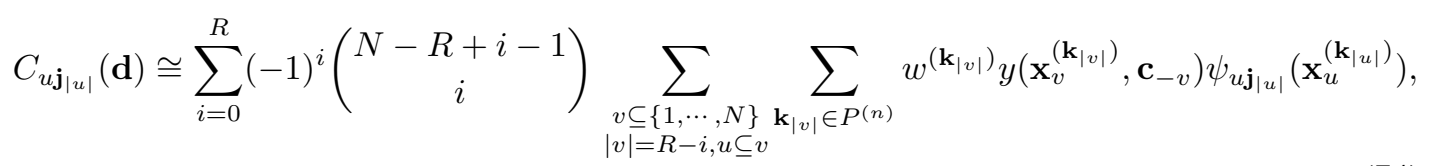

where $\mathbf{x}_{v}^{\left(\mathbf{k}_{|v|}\right)}=\left\{x_{i_{1}}^{\left(k_{1}\right)}, \cdots, x_{i_{|v|}}^{\left(k_{|v|}\right)}\right\}$ and $w^{\left(\mathbf{k}_{|v|}\right)}=\prod_{p=1}^{p=|v|} w_{i_{p}}^{\left(k_{p}\right)}$ is the product of integration weights generated by the variables indexed by the elements of $v$. Similarly, the coefficients $z_{\emptyset}(\mathbf{d})$ and $\bar{C}_{u \mathbf{j}|u|}(\mathbf{d})$ of an $\bar{S}$-variate, $\bar{m}$ th-order PDD approximation of $\tilde{y}_{S, m}^{r}(\mathbf{X})$, required in Option II for obtaining higher-order moments and their sensitivities, can also be estimated from the dimensionreduction integration. For independent coordinates of $\mathbf{X}$, as assumed here, a univariate Gauss quadrature rule is commonly used, where the integration points and associated weights depend on the probability distribution of $X_{i}$. They are readily available, for example, the Gauss-Hermite or Gauss-Legendre quadrature rule, when $X_{i}$ follows Gaussian or uniform distribution. For an arbitrary probability distribution of $X_{i}$, the Stieltjes procedure can be employed to generate the measureconsistent Gauss quadrature formulae [16, 17]. An $n$-point Gauss quadrature rule exactly integrates a polynomial with a total degree of at most $2 n-1$.

\subsection{Computational Expense}

The $S$-variate, $m$ th-order PDD approximation requires evaluations of $\sum_{k=0}^{k=S}\left(\begin{array}{c}N \\ k\end{array}\right) m^{k}$ expansion coefficients, including $y_{\emptyset}(\mathbf{d})$. If these coefficients are estimated by dimension-reduction integration 
with $R=S<N$ and, therefore, involve at most an $S$-dimensional tensor product of an $n$ point univariate quadrature rule depending on $m$, then the total cost for the $S$-variate, $m$ th-order approximation entails a maximum of $\sum_{k=0}^{k=S}\left(\begin{array}{l}N \\ k\end{array}\right) n^{k}(m)$ function evaluations. If the integration points include a common point in each coordinate - a special case of symmetric input probability density functions and odd values of $n-$ the number of function evaluations reduces to $\sum_{k=0}^{k=S}\left(\begin{array}{l}N \\ k\end{array}\right)(n(m)-1)^{k}$. Nonetheless, the computational complexity of the $S$-variate PDD approximation is an $S$ th-order polynomial with respect to the number of random variables or integration points. Therefore, PDD with dimension-reduction integration of the expansion coefficients alleviates the curse of dimensionality to an extent determined by $S$.

\section{NUMERICAL EXAMPLES}

Six numerical examples, comprising various mathematical functions and solid-mechanics problems, are illustrated to examine the accuracy, efficiency, and convergence properties of the PDD methods developed for calculating the first-order sensitivities of statistical moments, probability distributions, and reliability. The PDD expansion coefficients were estimated by dimension-reduction integration with the mean input as the reference point, $R=S$, and $n=m+1$, where $S$ and $m$ vary depending on the problem. In all examples, orthonormal polynomials and associated Gauss quadrature rules consistent with the probability distributions of input variables, including classical forms, if they exist, were employed. The first three examples entail independent and identical random variables, where $d_{k}$ is a distribution parameter of all random variables, whereas the last three examples contain merely independent random variables, where $d_{k}$ is a distribution parameter of a single random variable. The sample size for the embedded simulation of the PDD-MCS method is $10^{6}$ in Examples 2 and 3, and $10^{7}$ in Example 5. Whenever possible, the exact sensitivities were applied to verify the proposed methods, as in Examples 1 and 3. However, in Examples 2, 4, and 5, which do not support exact solutions, the benchmark results were generated from at least one of two crude MCS-based approaches: (1) crude MCS in conjunction with score functions (crude MCS/SF), which requires sampling of both the original function $y$ and the score function $s_{d_{k}}^{(1)}$; and (2) crude MCS in tandem with one-percent perturbation of finite-difference analysis (crude MCS/FD), which entails sampling of the original function $y$ only. The sample size for either version of the crude MCS is $10^{6}$ in Examples 2, 3, and 4, and $10^{7}$ in Example 5. The derivatives of log-density functions associated with the five types of random variables used in all examples are described in Table II.

\subsection{Example 1: A Trigonometric-Polynomial Function}

Consider the function

$$
y(\mathbf{X})=\mathbf{a}_{1}^{T} \mathbf{X}+\mathbf{a}_{2}^{T} \sin \mathbf{X}+\mathbf{a}_{3}^{T} \cos \mathbf{X}+\mathbf{X}^{T} \mathbf{M X},
$$

introduced by Oakley and O'Hagan [25], where $\mathbf{X}=\left\{X_{1}, \cdots, X_{15}\right\}^{T} \in \mathbb{R}^{15}$ is a 15-dimensional Gaussian input vector with mean vector $\mathbb{E}[\mathbf{X}]=\{\mu, \cdots, \mu\}^{T} \in \mathbb{R}^{15}$ and covariance matrix $\mathbb{E}\left[\mathbf{X X}^{T}\right]=\sigma^{2} \operatorname{diag}[1, \cdots, 1]=: \sigma^{2} \mathbf{I} \in \mathbb{R}^{15 \times 15} ; \mathbf{d}=\{\mu, \sigma\}^{T} ; \sin \mathbf{X}:=\left\{\sin X_{1}, \cdots, \sin X_{15}\right\}^{T} \in$ $\mathbb{R}^{15}$ and $\cos \mathbf{X}:=\left\{\cos X_{1}, \cdots, \cos X_{15}\right\}^{T} \in \mathbb{R}^{15}$ are compact notations for 15 -dimensional vectors of sine and cosine functions, respectively; and $\mathbf{a}_{i} \in \mathbb{R}^{15}, i=1,2,3$, and $\mathbf{M} \in \mathbb{R}^{15 \times 15}$ are coefficient vectors and matrix, respectively, obtained from Oakley and O'Hagan's paper [25]. The objective of this example is to evaluate the accuracy of the proposed PDD approximation in calculating the sensitivities of the first two moments, $m^{(1)}(\mathbf{d}):=\mathbb{E}_{\mathbf{d}}[y(\mathbf{X})]$ and $m^{(2)}(\mathbf{d}):=\mathbb{E}_{\mathbf{d}}\left[y^{2}(\mathbf{X})\right]$, with respect to the mean $\mu$ and standard deviation $\sigma$ of $X_{i}$ at $\mathbf{d}_{0}=\{0,1\}^{T}$.

Figures 1(a) through 1(d) present the plots of the relative errors in the approximate sensitivities, $\partial \tilde{m}_{S, m}^{(1)}\left(\mathbf{d}_{0}\right) / \partial \mu, \partial \tilde{m}_{S, m}^{(1)}\left(\mathbf{d}_{0}\right) / \partial \sigma, \partial \tilde{m}_{S, m}^{(2)}\left(\mathbf{d}_{0}\right) / \partial \mu$, and $\partial \tilde{m}_{S, m}^{(2)}\left(\mathbf{d}_{0}\right) / \partial \sigma$, obtained by the proposed univariate and bivariate PDD methods (Equations 55 and 56 for increasing orders of orthonormal polynomials, that is, when the PDD truncation parameters $S=1$ and $2,1 \leq m \leq 8$, and $m^{\prime}=2$. The measure-consistent Hermite polynomials and associated Gauss-Hermite quadrature rule were 
Table II. Derivatives of log-density functions for various probability distributions

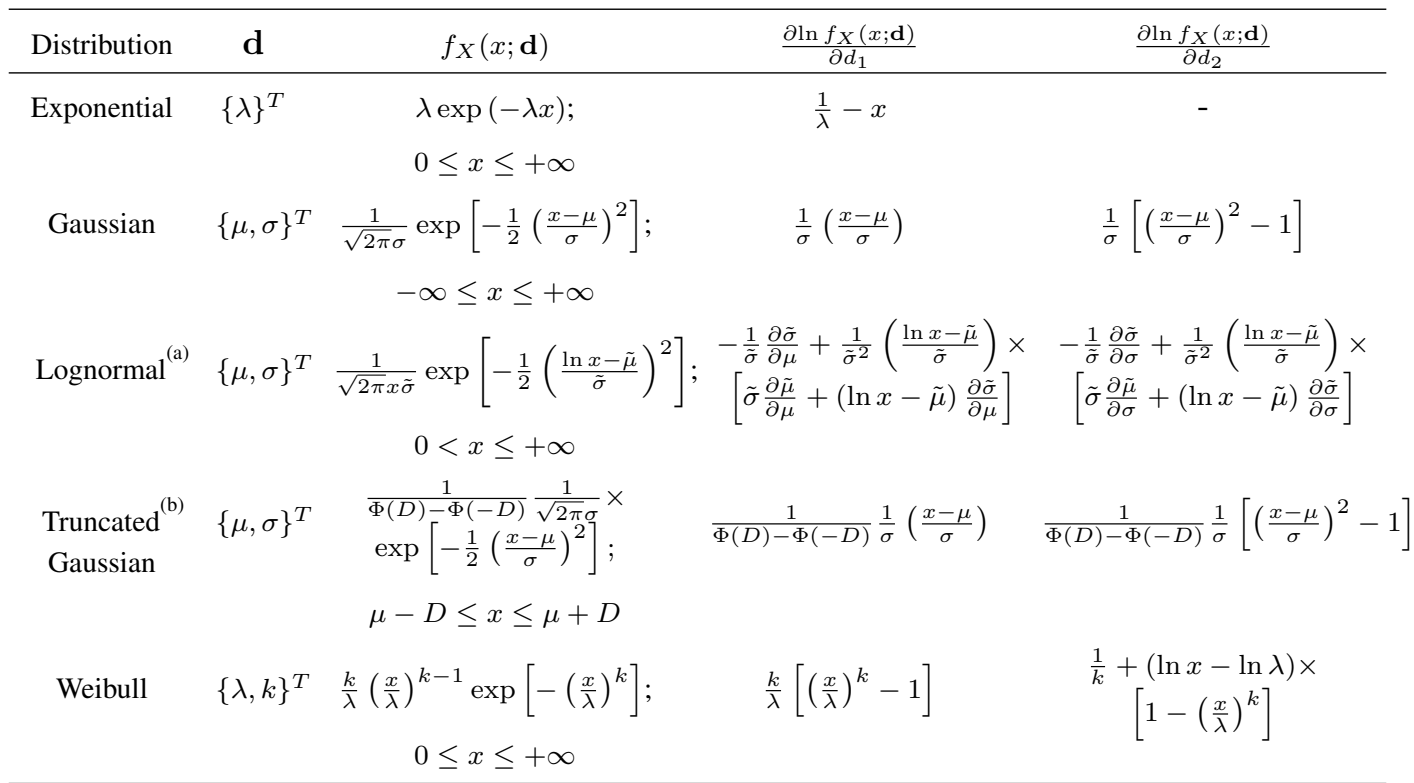

(a) $\tilde{\sigma}^{2}=\ln \left(1+\sigma^{2} / \mu^{2}\right)$ and $\tilde{\mu}=\ln \mu-\tilde{\sigma}^{2} / 2$. The partial derivatives of $\tilde{\mu}$ and $\tilde{\sigma}$ with respect to $\mu$ or $\sigma$ can be easily obtained, so they are not reported here.

(b) $\Phi(\cdot)$ is the cumulative distribution function of a standard Gaussian variable; $D>0$ is a constant.

used. The relative error is defined as the ratio of the absolute difference between the exact and approximate sensitivities, divided by the exact sensitivity, where the exact sensitivity can be easily calculated for the function $y$ in Equation 75. Although $y$ is a bivariate function of $\mathbf{X}$, the sensitivities of the first moment by the univariate and bivariate PDD approximations are identical for any $m$. This is because the expectations of $\tilde{y}_{1, m}(\mathbf{X})$ and $\tilde{y}_{2, m}(\mathbf{X})$, when $\mathbf{X}$ comprises independent variables, are the same function of $\boldsymbol{d}$. In this case, the errors committed by both PDD approximations drop at the same rate, as depicted in Figures 1(a) and 1 (b), resulting in rapid convergence of the sensitivities of the first moment. However, the same condition does not hold true for the sensitivities of the second moment, because the univariate and bivariate PDD approximations yield distinct sets of results. Furthermore, the errors in the sensitivities of the second moment by the univariate PDD approximation do not decay strictly monotonically, leveling off when $m$ crosses a threshold, as displayed in Figures 1. (c) and 1.(d). In contrast, the errors in the sensitivities of the second moment by the bivariate PDD approximation attenuate continuously with respect to $m$, demonstrating rapid convergence of the proposed solutions. The numerical results presented are consistent with the mean-square convergence of the sensitivities described by Proposition 6

\subsection{Example 2 : A Cubic Polynomial Function}

The second example is concerned with calculating the sensitivities of the probability distribution of

$$
y(\mathbf{X})=500-\left(X_{1}+X_{2}\right)^{3}+X_{1}-X_{2}-X_{3}+X_{1} X_{2} X_{3}-X_{4},
$$

where $X_{i}, i=1,2,3,4$, are four independent and identically distributed random variables. The sensitivities were calculated by the proposed PDD-MCS method using two approaches: (1) a direct approach employing measure-consistent orthonormal polynomials as bases and corresponding Gauss type quadrature rules for calculating the PDD expansion coefficients, and (2) an indirect approach transforming original random variables into Gaussian random variables, followed by Hermite orthonormal polynomials as bases and the Gauss-Hermite quadrature rule for calculating the expansion coefficients. Since Equation 76 represents a third-order polynomial, the measureconsistent orthonormal polynomials with the largest order $m=3$ should exactly reproduce $y$. In 


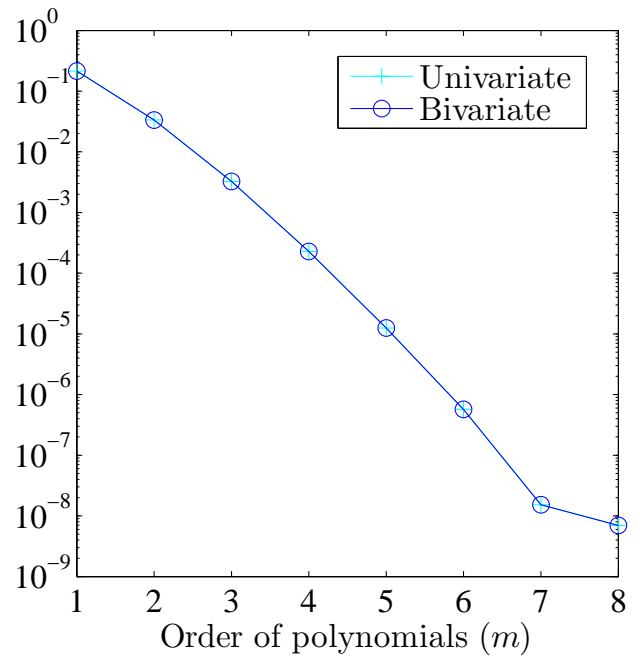

(a)

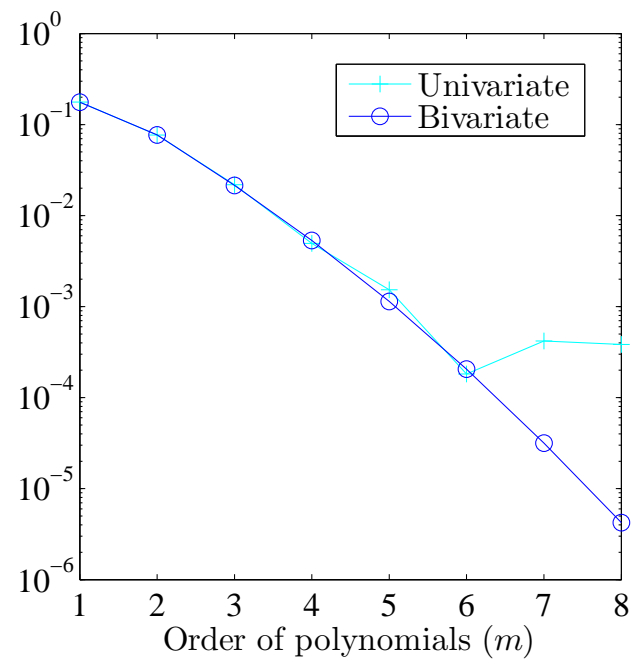

(c)

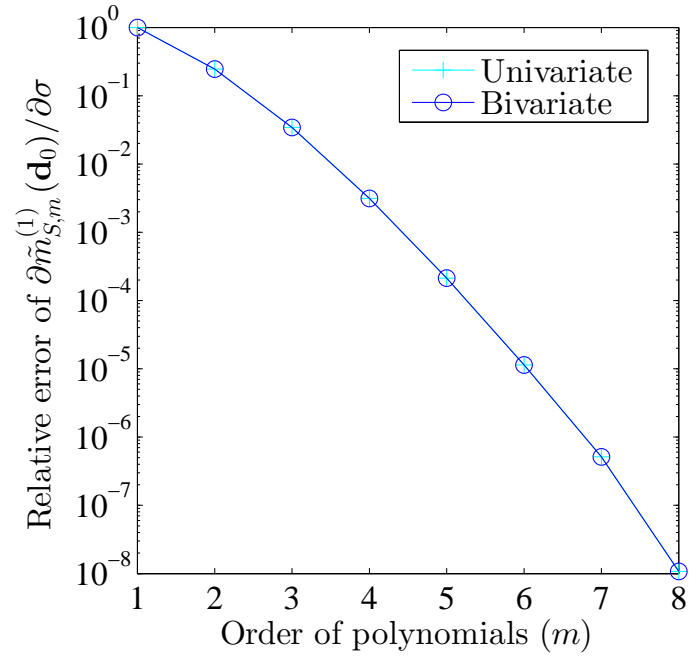

(b)

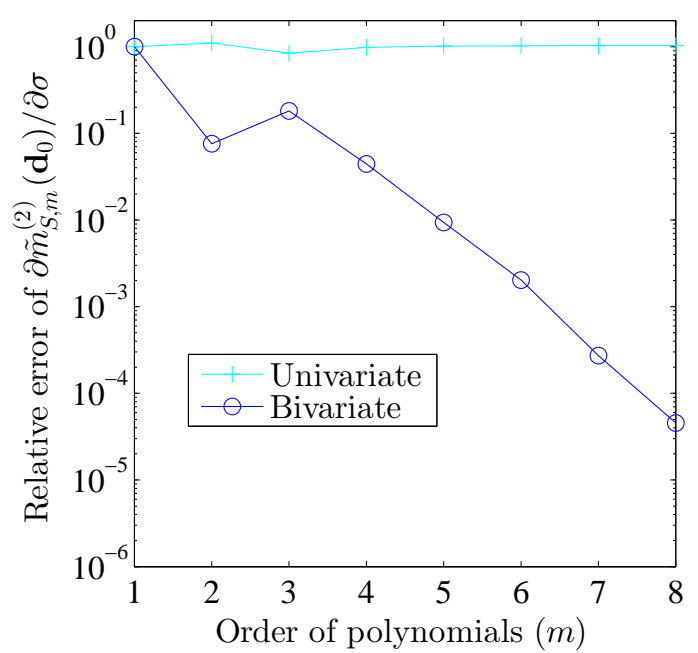

(d)

Figure 1. Relative errors in calculating the sensitivities of the first two moments of $y$ due to various PDD truncations; (a) $\partial \tilde{m}_{S, m}^{(1)}\left(\mathbf{d}_{0}\right) / \partial \mu$; (b) $\partial \tilde{m}_{S, m}^{(1)}\left(\mathbf{d}_{0}\right) / \partial \sigma ;$ (c) $\partial \tilde{m}_{S, m}^{(2)}\left(\mathbf{d}_{0}\right) / \partial \mu ;$ (d) $\partial \tilde{m}_{S, m}^{(2)}\left(\mathbf{d}_{0}\right) / \partial \sigma$ (Example 1)

which case, the highest order of integrands for calculating the PDD expansion coefficients is six; therefore, a four-point $(n=4)$ measure-consistent Gauss quadrature should provide exact values of the coefficients. In the direct approach, univariate $(S=1)$, bivariate $(S=2)$, and trivariate $(S=3)$ PDD approximations were applied, where the expansion coefficients were calculated using $R=S$, $m=3$, and $n=4$ in Equations 73 and 74. Therefore, the only source of error in a truncated PDD is the selection of $S$. In the indirect approach, the transformation of $y$, if the input variables follow non-Gaussian probability distributions, leads to non-polynomials in the space of Gaussian variables; therefore, approximation in a truncated PDD occur not only due to $S$, but also due to $m$. Hence several values of $3 \leq m \leq 6$ were employed for mappings into Gaussian variables. The coefficients in the indirect approach were calculated by the $n$-point Gauss-Hermite quadrature rule, where $n=m+1$.

A principal objective of this example is to gain insights on the choice of orthonormal polynomials for solving this problem by PDD approximations. Two distinct cases, depending on the probability distribution of input variables, were studied. 
6.2.1. Case 1: Exponential Distributions For exponential distributions of input random variables, the PDF

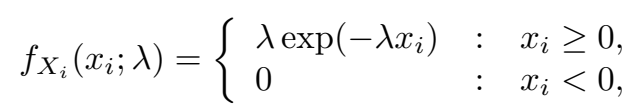

where $\lambda>0$ is the sole distribution parameter, $\mathbf{d}=\{\lambda\} \in \mathbb{R}$, and $\mathbf{d}_{0}=\{1\}$.

Figure 2(a) presents the sensitivities of the probability distribution of $y(\mathbf{X})$ with respect to $\lambda$ calculated at $\mathbf{d}_{0}$ for different values of $\xi$ by the direct approach. It contains four plots: one obtained from crude MCS/SF ( $10^{6}$ samples) and the remaining three generated from univariate $(S=1)$, bivariate $(S=2)$, and trivariate ( $S=3$ ) PDD-MCS methods. For the PDD-MCS methods, the measure-consistent Laguerre polynomials and associated Gauss-Laguerre quadrature rule were used. The sensitivity of distributions, all obtained for $m=3$, converge rapidly with respect to $S$. Compared with crude MCS/SF, the univariate PDD-MCS method is less accurate than others. This is due to the absence of cooperative effects of random variables in the univariate approximation. The bivariate PDD-MCS solution, which captures cooperative effects of any two variables, is remarkably close to the crude Monte Carlo results. The results from the trivariate decomposition and crude MCS/SF are coincident, as $\tilde{y}_{3,3}(\mathbf{X})$ is identical to $y(\mathbf{X})$, which itself is a trivariate function.

Using the indirect approach, Figures 2 b), 2(c), and 2(d) depict the sensitivities of the distribution of $y(\mathbf{X})$ by the univariate, bivariate, and trivariate PDD-MCS methods for several values of $m$, calculated when the original variables are transformed into standard Gaussian variables. The sensitivities obtained by all three decomposition methods from the indirect approach converge to the respective solutions from the direct approach when $m$ and $n$ increase. However, the lowest order of Hermite polynomials required to converge in the indirect approach is six, a number twice that employed in the direct approach employing Laguerre polynomials. This is due to higher nonlinearity of the mapped $y$ induced by the transformation from exponential to Gaussian variables. Clearly, the direct approach employing Laguerre polynomials and the Gauss-Laguerre quadrature rule is the preferred choice for calculating sensitivities of the probability distribution by the PDD-MCS method.

6.2.2. Case 2: Weibull Distributions For Weibull distributions of input random variables, the PDF

$$
f_{X_{i}}\left(x_{i} ; \lambda, k\right)= \begin{cases}\frac{k}{\lambda}\left(\frac{x_{i}}{\lambda}\right)^{k-1} \exp \left[-\left(\frac{x_{i}}{\lambda}\right)^{k}\right] & : \quad x_{i} \geq 0 \\ 0 & : \quad x_{i}<0\end{cases}
$$

where $\lambda>0$ and $k>0$ are scale and shape distribution parameters, respectively, $\mathbf{d}=\{\lambda, k\}^{T} \in \mathbb{R}^{2}$, and $\mathbf{d}_{0}=\{1,0.5\}^{T}$.

The sensitivities of the probability distribution of $y(\mathbf{X})$ with respect to $\lambda$ and $k$, calculated by the direct approach, at $\mathbf{d}_{0}$ is exhibited in Figures 3(a) and 4(a), respectively. Again, four plots, comprising the solutions from crude MCS/SF ( $10^{6}$ samples) and three PDD-MCS methods using the direct approach, are illustrated. Since classical orthonormal polynomials do not exist for Weibull probability measures, the Stieltjes procedure was employed to numerically determine the measureconsistent orthonormal polynomials and corresponding Gauss quadrature formula [16]. Similar to Case 1, both sensitivities of the distribution by the PDD-MCS method in Figures 3(a) and 4(a), all obtained for $m=3$, converge rapidly to crude MCS solutions with respect to $S$. However, the sensitivities of the distribution by all three PDD-MCS approximations, when calculated using the indirect approach and shown in Figures 3 (b) through 3(d) and Figures 4(b) through 4(d), fail to get closer even when the order of Hermite polynomials is twice that employed in the direct approach. The lack of convergence is attributed to a significantly higher nonlinearity of the transformation from Weibull to Gaussian variables than that from exponential to Gaussian variables. Therefore, a direct approach entailing measure-consistent orthogonal polynomials and associated Gauss quadrature rule, even in the absence of classical polynomials, is desirable for generating both accurate and efficient solutions by the PDD-MCS method. 


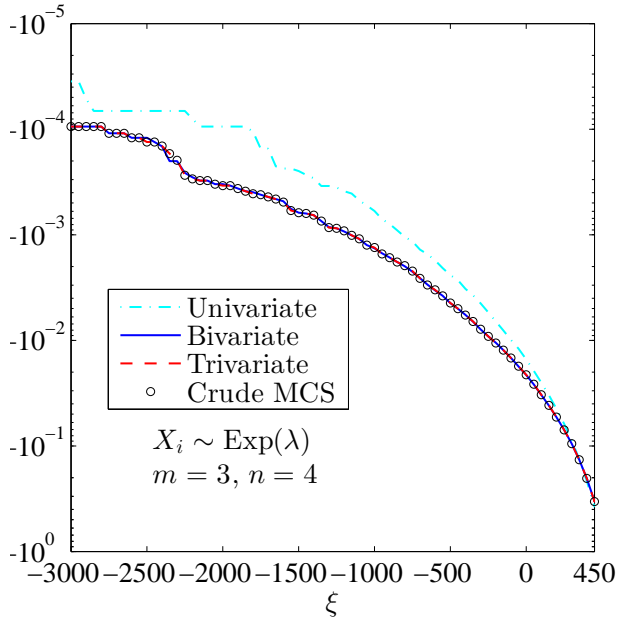

(a)

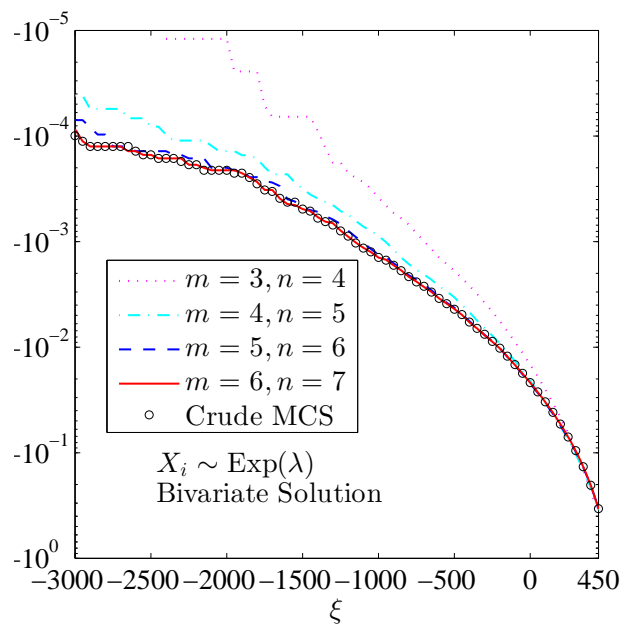

(c)

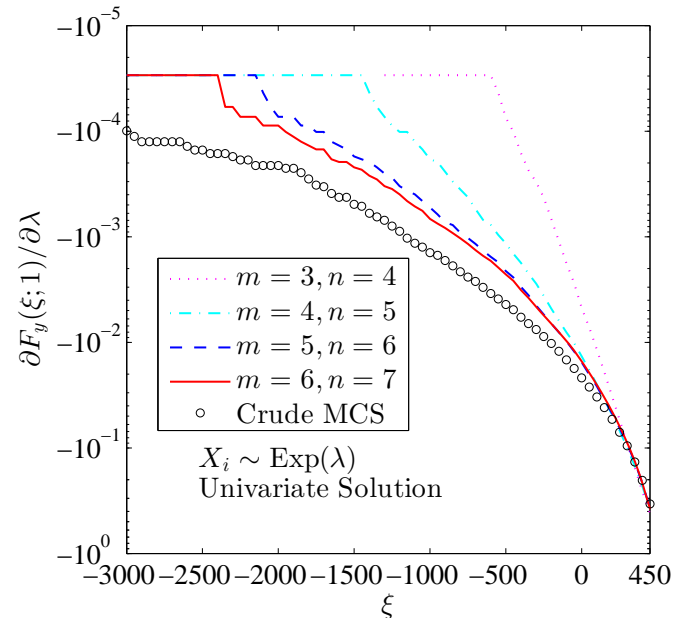

(b)

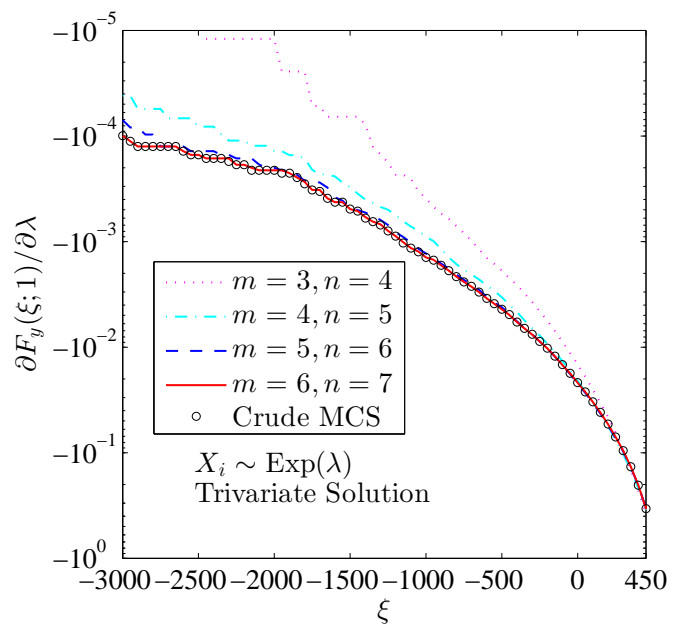

(d)

Figure 2. Sensitivities of the probability distribution of $y$ with respect to $\lambda$ for exponential distributions of input variables; (a) direct approach; (b) indirect approach-univariate; (c) indirect approach-bivariate; (d) indirect approach-trivariate (Example 2)

\subsection{Example 3: A Function of Gaussian Variables}

Consider a component reliability problem with the performance function

$$
y(\mathbf{X})=\frac{1}{1000+\sum_{i=1}^{N} X_{i}}-\frac{1}{1000+3 \sqrt{N}},
$$

where $\mathbf{X} \sim N(\boldsymbol{\mu}, \boldsymbol{\Sigma})$ is an $N$-dimensional Gaussian random vector with mean vector $\boldsymbol{\mu}=$ $\{\mu, \cdots, \mu\}^{T}$ and covariance matrix $\boldsymbol{\Sigma}=\sigma^{2} \operatorname{diag}[1, \cdots, 1]=: \sigma^{2} \mathbf{I}$, and $\mathbf{d}=\{\mu, \sigma\}^{T}$. The objective of this example is to evaluate the accuracy of the proposed PDD-SPA and PDD-MCS methods in calculating the failure probability $P_{F}(\mathbf{d}):=P_{\mathbf{d}}[y(\mathbf{X})<0]$ and its sensitivities $\partial P_{F}\left(\mathbf{d}_{0}\right) / \partial \mu$ and $\partial P_{F}\left(\mathbf{d}_{0}\right) / \partial \sigma$ at $\mathbf{d}_{0}=\{0,1\}^{T}$ for two problem sizes or dimensions: $N=10$ and $N=100$. The exact solutions for a general $N$-dimensional problem are

$$
P_{F}(\mathbf{d})=\Phi(-\beta), \frac{\partial P_{F}(\mathbf{d})}{\partial \mu}=\frac{\phi(-\beta) \sqrt{N}}{\sigma}, \frac{\partial P_{F}(\mathbf{d})}{\partial \sigma}=\frac{\phi(-\beta)(3-\mu \sqrt{N})}{\sigma^{2}},
$$




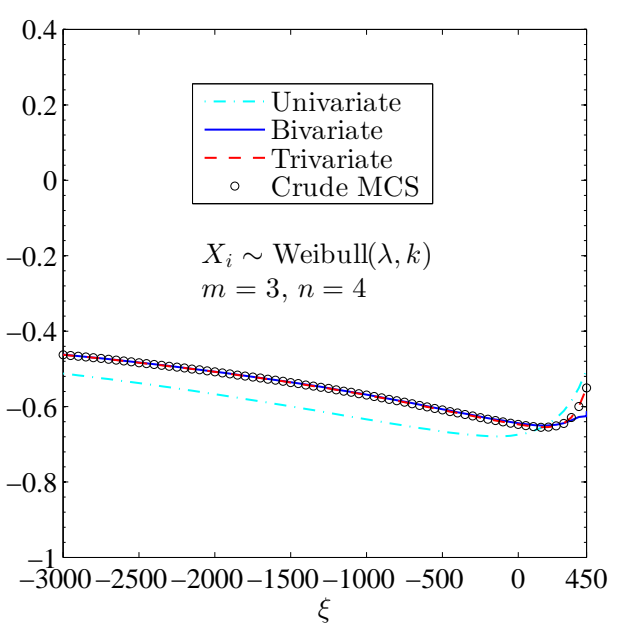

(a)

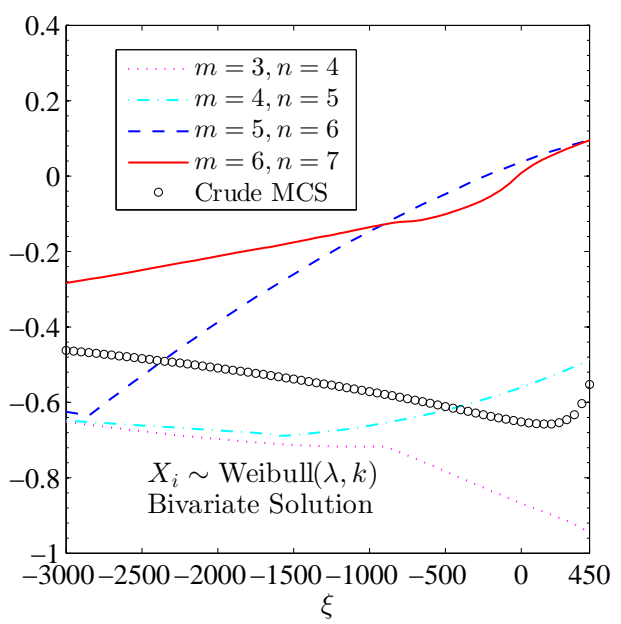

(c)

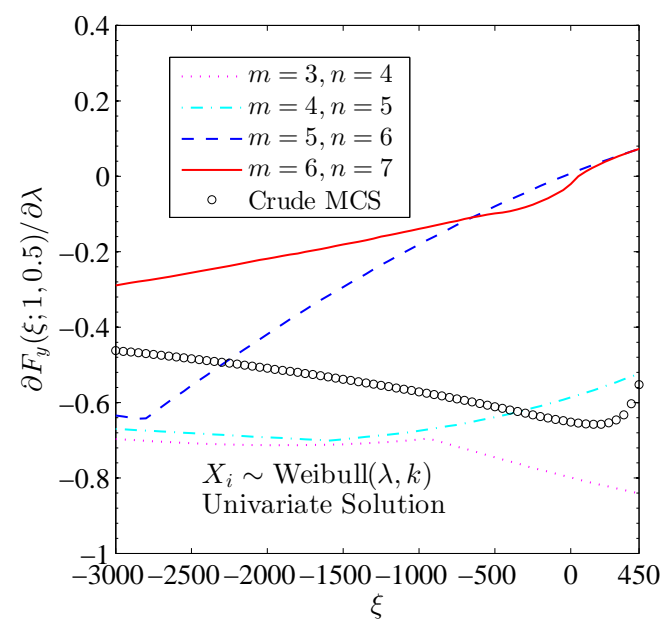

(b)

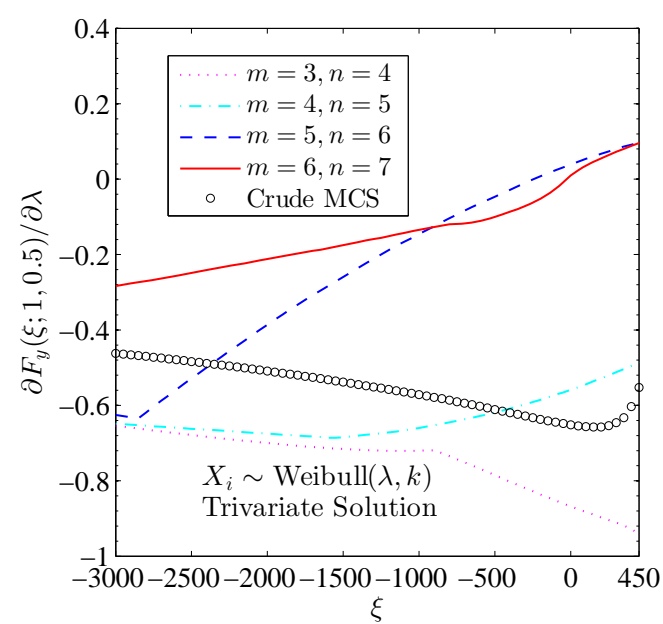

(d)

Figure 3. Sensitivities of the probability distribution of $y$ with respect to $\lambda$ for Weibull distributions of input variables; (a) direct approach; (b) indirect approach-univariate; (c) indirect approach-bivariate; (d) indirect approach-trivariate (Example 2)

where $\beta=(3-\mu \sqrt{N}) / \sigma$, provided that $0<\sigma^{2}<\infty$.

Since $y$ in Equation 79 is a non-polynomial function, the univariate $(S=1)$ or bivariate $(S=2)$ truncation of PDD for a finite value of $m$, regardless how large, provides only an approximation. Nonetheless, using only $m=3$ and $n=4$, the univariate and bivariate estimates of the failure probability and its two sensitivities by the PDD-SPA and PDD-MCS methods for $N=10$ are listed in Table III. The measure-consistent Hermite polynomials and associated Gauss-Hermite quadrature rule were used in both methods. The results of the PDD-SPA method are further broken down according to Options I (Equation 60) and II (Equation 61) for calculating all moments of order up to four to approximate the CGF of $y(\mathbf{X})$, as explained in Algorithm 1. Option I requires at most eightdimensional integrations in the bivariate PDD-SPA method for calculating the moments of $y(\mathbf{X})$, whereas Option II entails at most two-dimensional integrations for the values of $\bar{S}=2$ and $\bar{m}=6$ selected. However, the differences between the two respective estimates of the failure probability and its sensitivities by these options, in conjunction with either the univariate or the bivariate PDD approximation, are negligibly small. Therefore, Option II is not only accurate, but also facilitates efficient solutions by the PDD-SPA method, at least in this example. Compared with the results of crude MCS/SF ( $10^{6}$ samples) or the exact solution, also listed in Table III both univariate and 


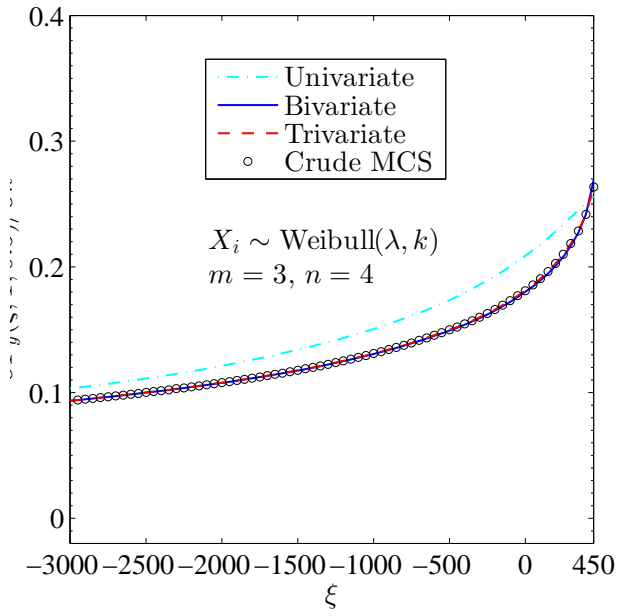

(a)

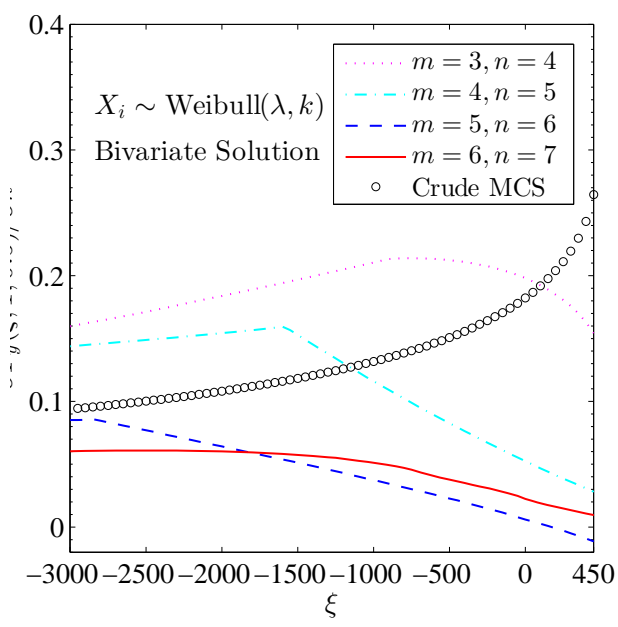

(c)

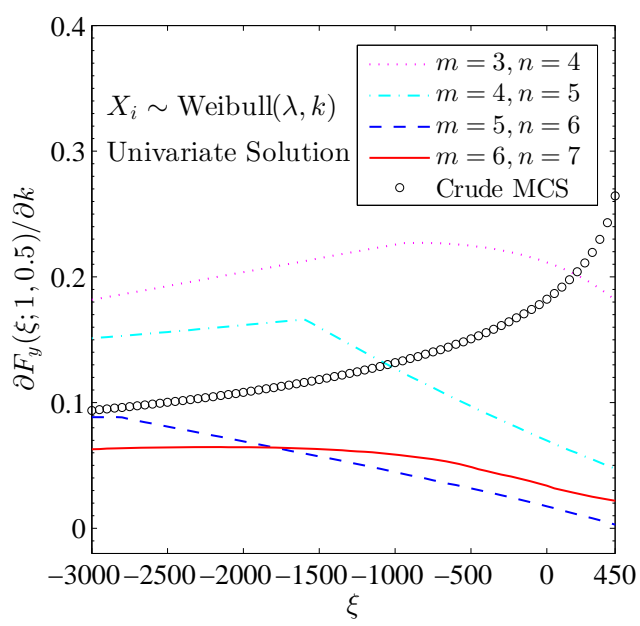

(b)

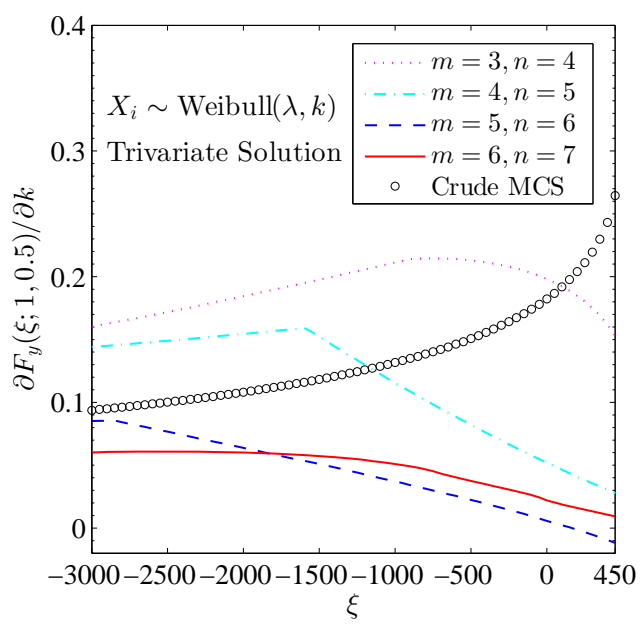

(d)

Figure 4. Sensitivities of the probability distribution of $y$ with respect to $k$ for Weibull distributions of input variables; (a) direct approach; (b) indirect approach-univariate; (c) indirect approach-bivariate; (d) indirect approach-trivariate (Example 2)

bivariate versions of the PDD-SPA method, regardless of the option, are satisfactory. The same trend holds for the univariate and bivariate PDD-MCS methods. No meaningful difference is found between the respective accuracies of the PDD-SPA and PDD-MCS solutions for a given truncation $S$. Indeed, the agreement between the bivariate solutions from the PDD-SPA or PDD-MCS method and the benchmark results is excellent.

For high-dimensional problems, such as $N=100$, Table IV summarizes the estimates of the failure probability and its sensitivities by the PDD-SPA and PDD-MCS methods using $m=3$. Due to the higher dimension, the PDD-SPA method with Option I requires numerous eight-dimensional integrations for calculating moments of $y(\mathbf{X})$ and is no longer practical. Therefore, the PDD-SPA method with Option II requiring only two-dimensional $(\bar{S}=2, \bar{m}=6)$ integrations was used for $N=100$. Again both univariate and bivariate approximations were invoked for the PDD-SPA and PDD-MCS methods. Compared with the benchmark results of crude MCS/SF ( $10^{6}$ samples) or the exact solution, listed in Table [V] the bivariate PDD-SPA method or the bivariate PDD-MCS method provides highly accurate solutions for this high-dimensional reliability problem.

Tables III and IV also specify the relative computational efforts of the PDD-SPA and PDDMCS methods, measured in terms of numbers of original function evaluations, when $N=10$ 
Table III. Component failure probability and sensitivities at $\mathbf{d}_{0}=\{0,1\}^{T}$ for $N=10$ (Example 3)

\begin{tabular}{ccccccccc}
\hline & $\begin{array}{c}\text { PDD-SPA } \\
\text { (Univariate, } \\
\text { Option I) }\end{array}$ & $\begin{array}{l}\text { PDD-SPA } \\
\text { (Univariate, } \\
\text { Option II) }\end{array}$ & $\begin{array}{c}\text { PDD-SPA } \\
\text { (Bivariate, } \\
\text { Option I) }\end{array}$ & $\begin{array}{c}\text { PDD-SPA } \\
\text { (Bivariate, } \\
\text { Option II) }\end{array}$ & $\begin{array}{c}\text { PDD-MCS } \\
\text { (Univariate) }\end{array}$ & $\begin{array}{c}\text { PDD-MCS } \\
\text { (Bivariate) }\end{array}$ & $\begin{array}{c}\text { Crude } \\
\text { MCS/SF }\end{array}$ & Exact \\
\hline $\begin{array}{c}P_{F}\left(\mathbf{d}_{0}\right) \\
\left(\times 10^{-3}\right)\end{array}$ & 1.349 & 1.453 & 1.349 & 1.347 & 1.510 & 1.397 & 1.397 & 1.350 \\
$\begin{array}{c}\partial P_{F}\left(\mathbf{d}_{0}\right) / \partial \mu \\
\left(\times 10^{-2}\right)\end{array}$ & 1.401 & 1.529 & 1.401 & 1.550 & 1.553 & 1.447 & 1.447 & 1.401 \\
$\begin{array}{c}\partial P_{F}\left(\mathbf{d}_{0}\right) / \partial \sigma \\
\left(\times 10^{-2}\right)\end{array}$ & 1.330 & 1.409 & 1.330 & 1.326 & 1.472 & 1.371 & 1.371 & 1.330 \\
$\quad \begin{array}{c}\text { No. of } \\
\text { function eval. }\end{array}$ & 41 & 41 & 761 & 761 & 41 & 761 & $10^{6}$ & - \\
\hline
\end{tabular}

Table IV. Component failure probability and sensitivities at $\mathbf{d}_{0}=\{0,1\}^{T}$ for $N=100$ (Example 3)

\begin{tabular}{ccccccc}
\hline & $\begin{array}{c}\text { PDD-SPA } \\
\text { (Univariate, } \\
\text { Option II) }\end{array}$ & $\begin{array}{c}\text { PDD-SPA } \\
\text { (Bivariate, } \\
\text { Option II) }\end{array}$ & $\begin{array}{c}\text { PDD-MCS } \\
\text { (Univariate) }\end{array}$ & $\begin{array}{c}\text { PDD-MCS } \\
\text { (Bivariate) }\end{array}$ & $\begin{array}{c}\text { Crude } \\
\text { MCS/SF }\end{array}$ & Exact \\
\hline $\begin{array}{c}P_{F}\left(\mathbf{d}_{0}\right) \\
\left(\times 10^{-3}\right)\end{array}$ & 1.731 & 1.320 & 1.724 & 1.344 & 1.352 & 1.350 \\
$\begin{array}{c}\partial P_{F}\left(\mathbf{d}_{0}\right) / \partial \mu \\
\left(\times 10^{-2}\right)\end{array}$ & 5.994 & 6.412 & 5.538 & 4.413 & 4.437 & 4.432 \\
$\begin{array}{c}\partial P_{F}\left(\mathbf{d}_{0}\right) / \partial \sigma \\
\left(\times 10^{-2}\right)\end{array}$ & 1.612 & 1.277 & 1.556 & 1.291 & 1.302 & 1.330 \\
$\begin{array}{c}\text { No. of } \\
\text { function eval. }\end{array}$ & 401 & 79,601 & 401 & 79,601 & 106 & - \\
\hline
\end{tabular}

and $N=100$. Given the truncation parameter $S$, the PDD-SPA and PDD-MCS methods require identical numbers of function evaluations, meaning that their computational costs are practically the same. Although the bivariate approximation is significantly more expensive than the univariate approximation, the former generates highly accurate solutions, as expected. However, both versions of the PDD-SPA or PDD-MCS method are markedly more economical than the crude MCS/SF method for solving this high-dimensional reliability problem.

\subsection{Example 4 : A Function of Non-Gaussian Variables}

Consider the univariate function [11]

$$
y(\mathbf{X})=X_{1}+2 X_{2}+2 X_{3}+X_{4}-5 X_{5}-5 X_{6}
$$

of six statistically independent and lognormally distributed random variables $X_{i}$ with means $\mu_{i}$ and standard deviations $c \mu_{i}, i=1, \cdots, 6$, where $c>0$ is a constant, representing the coefficient of variation of $X_{i}$. The design vector $\mathbf{d}=\left\{\mu_{1}, \cdots \mu_{6}, \sigma_{1}, \cdots, \sigma_{6}\right\}^{T}$. The objective of this example is to evaluate the accuracy of the proposed PDD-SPA method in estimating the failure probability $P_{F}(\mathbf{d}):=P_{\mathbf{d}}[y(\mathbf{X})<0]$ and its sensitivities $\partial P_{F}(\mathbf{d}) / \partial \mu_{i}$ and $\partial P_{F}(\mathbf{d}) / \partial \sigma_{i}, i=1, \cdots, 6$, at $\mathbf{d}=$ $\mathbf{d}_{0}=\{120,120,120,120,50,40,120 c, 120 c, 120 c, 120 c, 50 c, 40 c\}^{T}$ for $0.1 \leq c \leq 0.7$.

The function $y$, being both univariate and linear, is exactly reproduced by the univariate $(S=1)$, first-order $(m=1)$ PDD approximation when orthonormal polynomials consistent with lognormal probability measures are used. Therefore, the univariate, first-order PDD approximation, along with Option I (Equation 60), was employed in the PDD-SPA method to approximate $P_{F}\left(\mathbf{d}_{0}\right)$, $\partial P_{F}\left(\mathbf{d}_{0}\right) / \partial \mu_{i}$, and $\partial P_{F}\left(\mathbf{d}_{0}\right) / \partial \sigma_{i}$. All moments of order up to four were estimated according to Algorithm 11. The measure-consistent solutions by the PDD-SPA method and crude MCS/SF are presented in Figures 5(a), 5(b), and 5(c). Huang and Zhang [11], who solved the same 
problem, reported similar results, but at the expense of higher-order integrations stemming from transformation to Gaussian variables. No such transformation was required or performed in this work. According to Figure 5(a), the failure probability curve generated by the PDD-SPA method closely traces the path of crude MCS/SF ( $10^{6}$ samples) for low coefficients of variation, although a slight deviation begins to appear when $c$ exceeds about 0.4 . The loss of accuracy becomes more pronounced when comparing the sensitivities of the failure probability with respect to means and standard deviations in Figures 5(b) and 5(c). Indeed, for large coefficients of variation, that is, for $c>0.4$, some of the sensitivities are no longer accurately calculated by the PDD-SPA method. This is because the fourth-order $(Q=4)$ approximation of the CGF of $y(\mathbf{X})$, used for constructing the PDD-SPA method, is inadequate. Indeed, Table $\mathrm{V}$ reveals that the relative errors in the fourthorder Taylor approximation of the CGF, obtained by MCS ( $10^{8}$ samples) and evaluated at respective saddlepoints, rises with increasing values of the coefficient of variation from 0.2 to 0.7 . Therefore, a truncation larger than four is warranted for higher-order approximations of CGF, but doing so engenders an added difficulty in finding a unique saddlepoint. The topic merits further study.

Table V. Relative errors in calculating CGF (Example 4)

\begin{tabular}{ccc}
\hline$c$ & $t_{s}$ & Relative error $^{(\mathrm{a})}$ \\
\hline 0.1 & $-1.0029 \times 10^{-1}$ & 0.0248 \\
0.2 & $-2.5008 \times 10^{-2}$ & 0.0068 \\
0.3 & $-1.1066 \times 10^{-2}$ & 0.0125 \\
0.4 & $-6.1850 \times 10^{-3}$ & 0.0183 \\
0.5 & $-3.9250 \times 10^{-3}$ & 0.0329 \\
0.6 & $-2.6966 \times 10^{-3}$ & 0.0447 \\
0.7 & $-1.9551 \times 10^{-3}$ & 0.2781 \\
\hline
\end{tabular}

(a) The sample size of MCS is $10^{8}$.

It is important to note that the univariate, first-order PDD-MCS method, employing measureconsistent orthonormal polynomials, should render the same solution of crude MCS/SF. This is the primary reason why the PDD-MCS results are not depicted in Figures 5 (a) through 5 (c). Nonetheless, the PDD-MCS method should be more accurate than the PDD-SPA method in solving this problem, especially at larger coefficients of variation.

\subsection{Example 5: A Six-Bay, Twenty-One-Bar Truss}

This example demonstrates how system reliability and its sensitivities can be efficiently estimated with the PDD-MCS method. A linear-elastic, six-bay, twenty-one-bar truss structure, with geometric properties shown in Figure 6, is simply supported at nodes 1 and 12, and is subjected to four concentrated loads of $10,000 \mathrm{lb}(44,482 \mathrm{~N})$ at nodes 3, 5, 9, and 11 and a concentrated load of $16,000 \mathrm{lb}(71,172 \mathrm{~N})$ at node 7 . The truss material is made of an aluminum alloy with the Young's modulus $E=10^{7}$ psi (68.94 GPa). The random input is $\mathbf{X}=\left\{X_{1}, \cdots, X_{21}\right\}^{T} \in \mathbb{R}^{21}$, where $X_{i}$ is the cross-sectional areas of the $i$ th truss member. The random variables are independent and lognormally distributed with means $\mu_{i}, i=1, \cdots, 21$, each of which has a ten percent coefficient of variation. From linear-elastic finite-element analysis (FEA), the maximum vertical displacement $v_{\max }(\mathbf{X})$ and maximum axial stress $\sigma_{\max }(\mathbf{X})$ occur at node 7 and member 3 or 4 , respectively, where the permissible displacement and stress are limited to $d_{\text {allow }}=0.266 \mathrm{in}(6.76 \mathrm{~mm})$ and $\sigma_{\text {allow }}=37,680 \mathrm{psi}(259.8 \mathrm{MPa})$, respectively. The system-level failure set is defined as $\Omega_{F}:=\{\mathbf{x}$ : $\left.\left\{y_{1}(\mathbf{x})<0\right\} \cup\left\{y_{2}(\mathbf{x})<0\right\}\right\}$, where the performance functions

$$
y_{1}(\mathbf{X})=1-\frac{\left|v_{\max }(\mathbf{X})\right|}{d_{\text {allow }}}, y_{2}(\mathbf{X})=1-\frac{\left|\sigma_{\max }(\mathbf{X})\right|}{\sigma_{\text {allow }}} .
$$

The design vector is $\mathbf{d}=\left\{\mu_{1}, \cdots, \mu_{21}\right\}^{T}$. The objective of this example is to evaluate the accuracy of the proposed PDD-MCS method in estimating the system failure probability $P_{F}(\mathbf{d}):=$ 


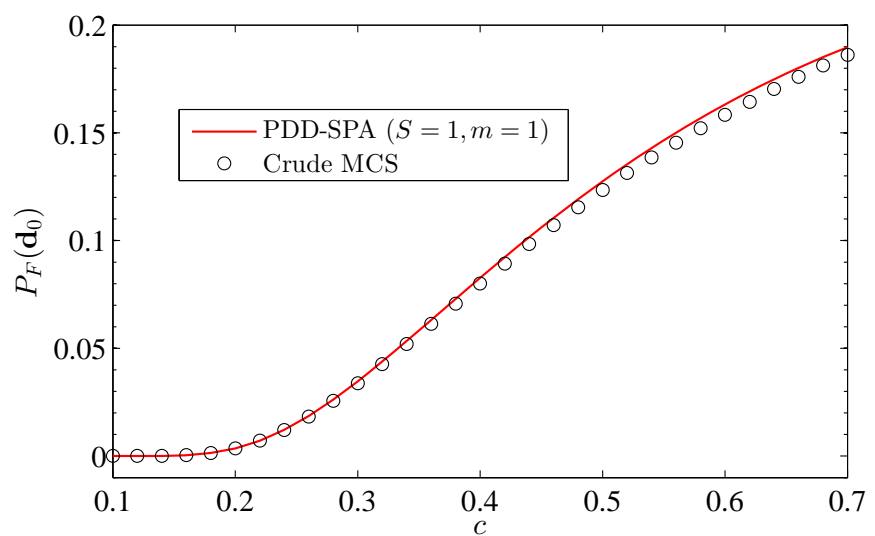

(a)

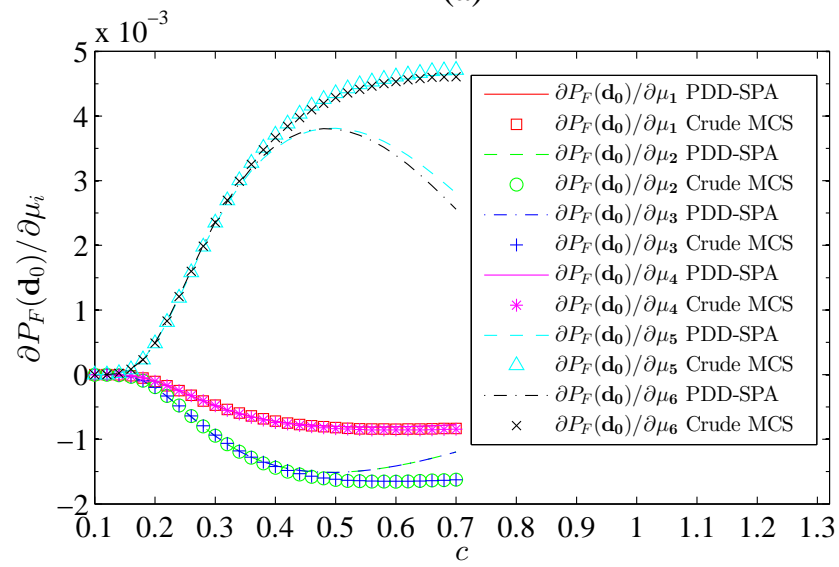

(b)

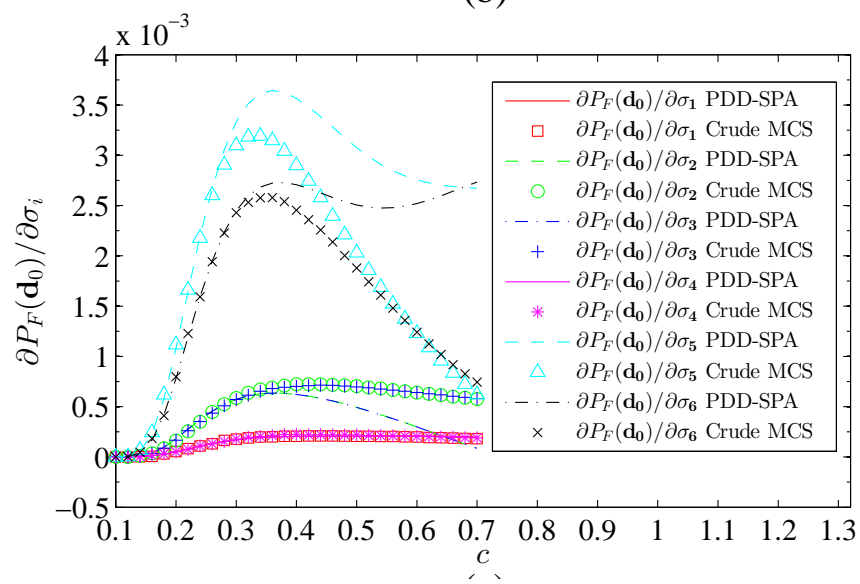

Figure 5. Results of the reliability and sensitivity analyses by the PDD-SPA method and crude MCS/SF; (a) failure probability; (b) sensitivities of failure probability with respect to means; (c) sensitivities of failure probability with respect to standard deviations (Example 4)

$P_{\mathbf{d}}\left[\left\{y_{1}(\mathbf{X})<0\right\} \cup\left\{y_{2}(\mathbf{X})<0\right\}\right]$ and its sensitivities $\partial P_{F}(\mathbf{d}) / \partial \mu_{i}, \quad i=1, \ldots, 21$ at $\mathbf{d}=\mathbf{d}_{0}=$ $\{2,2,2,2,2,2,10,10,10,10,10,10,3,3,3,3,3,1,1,1,1\}^{T}$ in $^{2}\left(\times 2.54^{2} \mathrm{~cm}^{2}\right)$.

Table/VI presents the system failure probability and its 21 sensitivities obtained using the bivariate $(S=2)$, third-order $(m=3)$ PDD approximations of $y_{1}(\mathbf{X})$ and $y_{2}(\mathbf{X})$ and two versions of crude MCS: crude MCS/SF and crude MCS/FD, providing benchmark solutions. The crude MCS/FD method does not depend on score functions and, therefore, facilitates an independent verification 


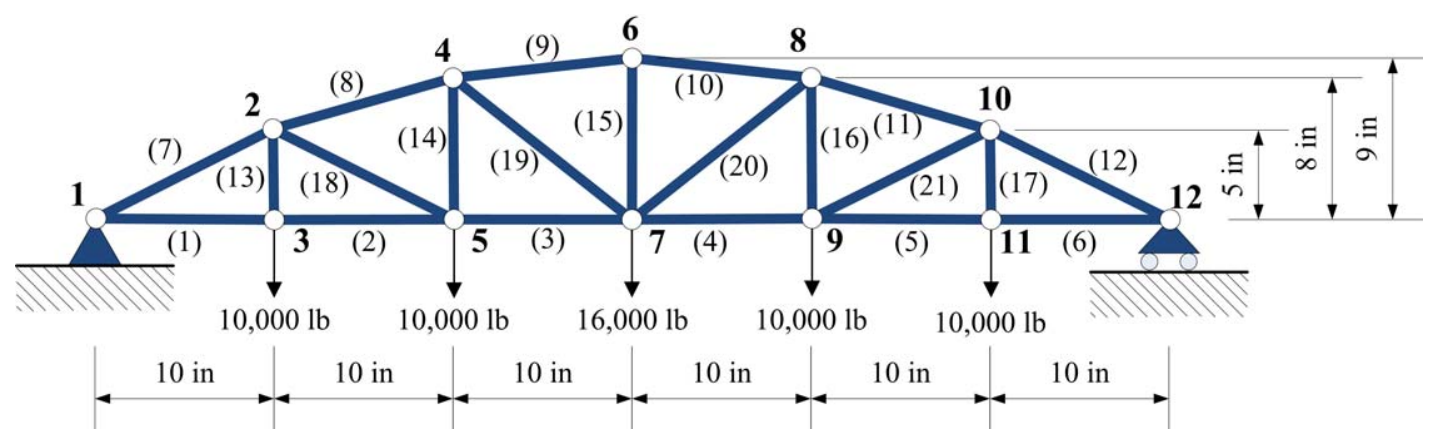

Figure 6. A six-bay, twenty-one-bar truss structure (Example 5)

of the PDD-MCS method. The respective sensitivities obtained by the PDD-MCS method and crude MCS/SF are practically the same. However, crude MCS/FD typically gives biased sensitivity estimates, where slight fluctuations in the results are expected due to a finite variance of the estimator. For two instances, such as when the sensitivities are too small, crude MCS/FD produces trivial solutions and hence cannot be used as reference solutions. Nonetheless, the general quality of agreement between the results of the PDD-MCS method and crude MCS/FD is very good. Comparing the computational efforts, only 3445 FEA were required to produce the results of the PDD-MCS method in Table VI, whereas $10^{7}$ and $22 \times 10^{7}$ FEA (samples) were incurred by crude MCS/SF and crude MCS/FD, respectively. The 22-fold increase in the number of FEA in crude MCS/FD is due to forward finite-difference calculations entailing all 21 sensitivities. Therefore, the PDD-MCS method provides not only highly accurate, but also vastly efficient, solutions of system reliability problems.

It is important to recognize that the PDD-SPA method can be applied to solve this seriessystem reliability problem by interpreting the failure domain as $\Omega_{F}:=\left\{\mathbf{x}: y_{s}(\mathbf{x})<0\right\}$, where $y_{s}(\mathbf{X}):=\min \left\{y_{1}(\mathbf{X}), y_{2}(\mathbf{X})\right\}$ and then constructing a PDD approximation of $y_{s}(\mathbf{X})$. In doing so, however, $y_{s}$ is no longer a smooth function of $\mathbf{X}$, meaning that the convergence properties of the PDD-SPA method can be significantly deteriorated. More importantly, the PDD-SPA method is not suitable for a general system reliability problem involving multiple, interdependent component performance functions. This is the primary reason why the results of the PDD-SPA method are not included in this example.

\subsection{Example 6: A Three-Hole Bracket}

The final example involves robust shape design optimization of a two-dimensional, three-hole bracket, where nine random shape parameters, $X_{i}, i=1, \cdots, 9$, describe its inner and outer boundaries, while maintaining symmetry about the central vertical axis. The design variables, $d_{k}=\mathbb{E}_{\mathbf{d}}\left[X_{k}\right], k=1, \cdots, 9$, are the means of these independent random variables, with Figure 7 (a) depicting the initial design of the bracket geometry at the mean values of the shape parameters. The bottom two holes are fixed, and a deterministic horizontal force $F=15,000 \mathrm{~N}$ is applied at the center of the top hole. The bracket material has a deterministic mass density $\rho=7810$ $\mathrm{kg} / \mathrm{m}^{3}$, deterministic elastic modulus $E=207.4 \mathrm{GPa}$, deterministic Poisson's ratio $\nu=0.3$, and deterministic uniaxial yield strength $S_{y}=800 \mathrm{MPa}$. The objective is to minimize the secondmoment properties of the mass of the bracket by changing the shape of the geometry such that the maximum von Mises stress $\sigma_{e, \max }(\mathbf{X})$ does not exceed the yield strength $S_{y}$ of the material with 
Table VI. System failure probability and sensitivities for the six-bay, twenty-one-bar truss (Example 5)

\begin{tabular}{ccrr}
\hline & PDD-MCS & Crude MCS/SF & Crude MCS/FD \\
\hline$P_{F}\left(\mathbf{d}_{0}\right)$ & $8.1782 \times 10^{-3}$ & $8.3890 \times 10^{-3}$ & $8.3890 \times 10^{-3}$ \\
$\partial P_{F}\left(\mathbf{d}_{0}\right) / \partial \mu_{1}$ & $-2.6390 \times 10^{-2}$ & $-2.6546 \times 10^{-2}$ & $-2.3895 \times 10^{-2}$ \\
$\partial P_{F}\left(\mathbf{d}_{0}\right) / \partial \mu_{2}$ & $-2.6385 \times 10^{-2}$ & $-2.6505 \times 10^{-2}$ & $-2.3810 \times 10^{-2}$ \\
$\partial P_{F}\left(\mathbf{d}_{0}\right) / \partial \mu_{3}$ & $-1.0010 \times 10^{-1}$ & $-1.0320 \times 10^{-1}$ & $-8.8875 \times 10^{-2}$ \\
$\partial P_{F}\left(\mathbf{d}_{0}\right) / \partial \mu_{4}$ & $-3.5684 \times 10^{-2}$ & $-3.5972 \times 10^{-2}$ & $-3.1960 \times 10^{-2}$ \\
$\partial P_{F}\left(\mathbf{d}_{0}\right) / \partial \mu_{5}$ & $-2.6356 \times 10^{-2}$ & $-2.6469 \times 10^{-2}$ & $-2.3825 \times 10^{-2}$ \\
$\partial P_{F}\left(\mathbf{d}_{0}\right) / \partial \mu_{6}$ & $-2.6266 \times 10^{-2}$ & $-2.6364 \times 10^{-2}$ & $-2.3950 \times 10^{-2}$ \\
$\partial P_{F}\left(\mathbf{d}_{0}\right) / \partial \mu_{7}$ & $-1.3189 \times 10^{-3}$ & $-1.3213 \times 10^{-3}$ & $-1.1970 \times 10^{-3}$ \\
$\partial P_{F}\left(\mathbf{d}_{0}\right) / \partial \mu_{8}$ & $-1.3294 \times 10^{-3}$ & $-1.3244 \times 10^{-3}$ & $-1.2820 \times 10^{-3}$ \\
$\partial P_{F}\left(\mathbf{d}_{0}\right) / \partial \mu_{9}$ & $-1.6665 \times 10^{-3}$ & $-1.6514 \times 10^{-3}$ & $-1.5610 \times 10^{-3}$ \\
$\partial P_{F}\left(\mathbf{d}_{0}\right) / \partial \mu_{10}$ & $-1.7554 \times 10^{-3}$ & $-1.7576 \times 10^{-3}$ & $-1.5670 \times 10^{-3}$ \\
$\partial P_{F}\left(\mathbf{d}_{0}\right) / \partial \mu_{11}$ & $-1.3892 \times 10^{-3}$ & $-1.3945 \times 10^{-3}$ & $-1.2530 \times 10^{-3}$ \\
$\partial P_{F}\left(\mathbf{d}_{0}\right) / \partial \mu_{12}$ & $-1.3136 \times 10^{-3}$ & $-1.3140 \times 10^{-3}$ & $-1.2060 \times 10^{-3}$ \\
$\partial P_{F}\left(\mathbf{d}_{0}\right) / \partial \mu_{13}$ & $9.1378 \times 10^{-5}$ & $7.2857 \times 10^{-5}$ & 0.0 \\
$\partial P_{F}\left(\mathbf{d}_{0}\right) / \partial \mu_{14}$ & $2.3126 \times 10^{-4}$ & $2.0942 \times 10^{-4}$ & $1.3000 \times 10^{-4}$ \\
$\partial P_{F}\left(\mathbf{d}_{0}\right) / \partial \mu_{15}$ & $-6.3125 \times 10^{-4}$ & $-6.2761 \times 10^{-4}$ & $-5.8333 \times 10^{-4}$ \\
$\partial P_{F}\left(\mathbf{d}_{0}\right) / \partial \mu_{16}$ & $2.2333 \times 10^{-4}$ & $2.2261 \times 10^{-4}$ & $1.3333 \times 10^{-4}$ \\
$\partial P_{F}\left(\mathbf{d}_{0}\right) / \partial \mu_{17}$ & $-3.0844 \times 10^{-5}$ & $-3.9551 \times 10^{-5}$ & 0.0 \\
$\partial P_{F}\left(\mathbf{d}_{0}\right) / \partial \mu_{18}$ & $-2.0729 \times 10^{-4}$ & $-2.6582 \times 10^{-4}$ & $-8.8000 \times 10^{-4}$ \\
$\partial P_{F}\left(\mathbf{d}_{0}\right) / \partial \mu_{19}$ & $-3.5881 \times 10^{-3}$ & $-3.4714 \times 10^{-3}$ & $-3.2900 \times 10^{-3}$ \\
$\partial P_{F}\left(\mathbf{d}_{0}\right) / \partial \mu_{20}$ & $-4.1604 \times 10^{-3}$ & $-4.0774 \times 10^{-3}$ & $-3.2200 \times 10^{-3}$ \\
$\partial P_{F}\left(\mathbf{d}_{0}\right) / \partial \mu_{21}$ & $-7.7002 \times 10^{-4}$ & $-7.2830 \times 10^{-4}$ & $-8.5000 \times 10^{-4}$ \\
No. of FEA & 3445 & 107 & $22 \times 10^{7}$ \\
\hline
\end{tabular}

$99.875 \%$ probability if $y_{1}$ is Gaussian. Mathematically, the RDO problem is defined to

$$
\begin{aligned}
\min _{\mathbf{d} \in \mathcal{D}} c_{0}(\mathbf{d})= & 0.5 \frac{\mathbb{E}_{\mathbf{d}}\left[y_{0}(\mathbf{X})\right]}{\mathbb{E}_{\mathbf{d}_{0}}\left[y_{0}(\mathbf{X})\right]}+0.5 \frac{\sqrt{\operatorname{var}_{\mathbf{d}}\left[y_{0}(\mathbf{X})\right]}}{\sqrt{\operatorname{var}_{\mathbf{d}_{0}}\left[y_{0}(\mathbf{X})\right]}}, \\
\text { subject to } c_{1}(\mathbf{d})= & 3 \sqrt{\operatorname{var}_{\mathbf{d}}\left[y_{1}(\mathbf{X})\right]}-\mathbb{E}_{\mathbf{d}}\left[y_{1}(\mathbf{X})\right] \leq 0, \\
& 0 \mathrm{~mm} \leq d_{1} \leq 14 \mathrm{~mm}, 17 \mathrm{~mm} \leq d_{2} \leq 35 \mathrm{~mm}, \\
& 10 \mathrm{~mm} \leq d_{3} \leq 30 \mathrm{~mm}, 30 \mathrm{~mm} \leq d_{4} \leq 40 \mathrm{~mm}, \\
& 12 \mathrm{~mm} \leq d_{5} \leq 30 \mathrm{~mm}, 12 \mathrm{~mm} \leq d_{6} \leq 30 \mathrm{~mm}, \\
& 50 \mathrm{~mm} \leq d_{7} \leq 140 \mathrm{~mm},-15 \mathrm{~mm} \leq d_{8} \leq 10 \mathrm{~mm}, \\
& -8 \mathrm{~mm} \leq d_{9} \leq 15 \mathrm{~mm},
\end{aligned}
$$

where $\mathbf{d}=\left(d_{1}, \cdots, d_{9}\right) \in \mathcal{D} \subset \mathbb{R}^{9}$ is the design vector;

$$
y_{0}(\mathbf{X})=\rho \int_{\mathcal{D}^{\prime}(\mathbf{X})} d \mathcal{D}^{\prime}
$$

and

$$
y_{1}(\mathbf{X})=S_{y}-\sigma_{e, \max }(\mathbf{X})
$$

are two random response functions; $\mathbb{E}_{\mathbf{d}}\left[y_{0}(\mathbf{X})\right]$ and $\operatorname{var}_{\mathbf{d}}\left[y_{0}(\mathbf{X})\right]:=\mathbb{E}_{\mathbf{d}}\left[y_{0}(\mathbf{X})-\mathbb{E}_{\mathbf{d}}\left[y_{0}(\mathbf{X})\right]\right]^{2}$ are the mean and variance, respectively, of $y_{0}$ at design $\mathbf{d}$; and $\mathbb{E}_{\mathbf{d}}\left[y_{1}(\mathbf{X})\right]$ and $\operatorname{var}_{\mathbf{d}}\left[y_{1}(\mathbf{X})\right]:=$ $\mathbb{E}_{\mathbf{d}}\left[y_{1}(\mathbf{X})-\mathbb{E}_{\mathbf{d}}\left[y_{1}(\mathbf{X})\right]\right]^{2}$ are the mean and variance, respectively, of $y_{1}$ at design $\mathbf{d}$. The initial design $\mathbf{d}_{0}=\{0,30,10,40,20,20,75,0,0\}^{T} \mathrm{~mm}$. Figure 7 (b) portrays the contours of the von Mises stress calculated by the FEA of the initial bracket design, which comprises 11,908 nodes and 3914 
eight-noded quadrilateral elements. A plane stress condition was assumed. The approximate optimal solution is denoted by $\tilde{\mathbf{d}}^{*}=\left\{\tilde{d}_{1}^{*}, \cdots, \tilde{d}_{9}^{*}\right\}^{T}$.

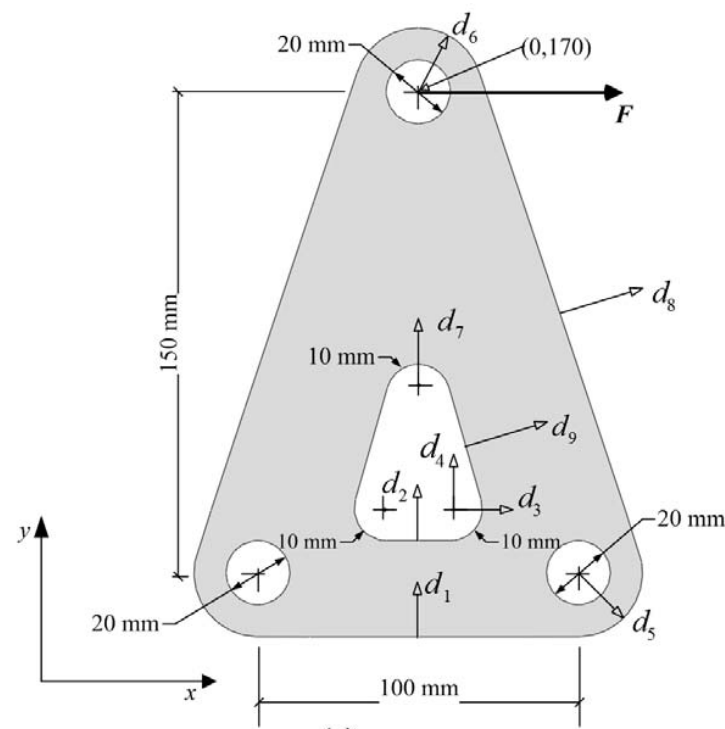

(a)

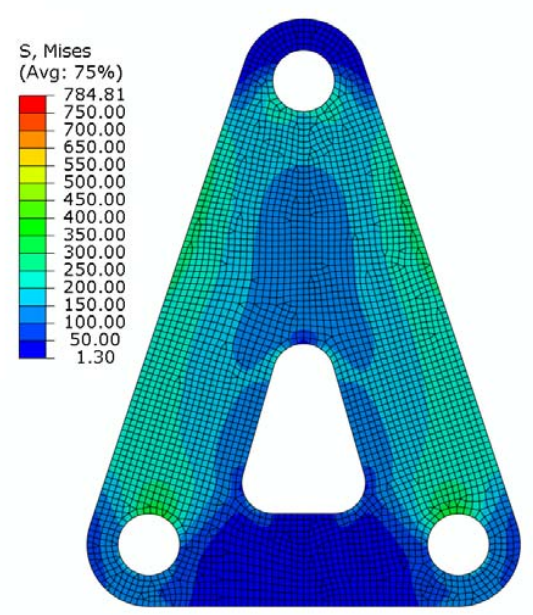

(b)

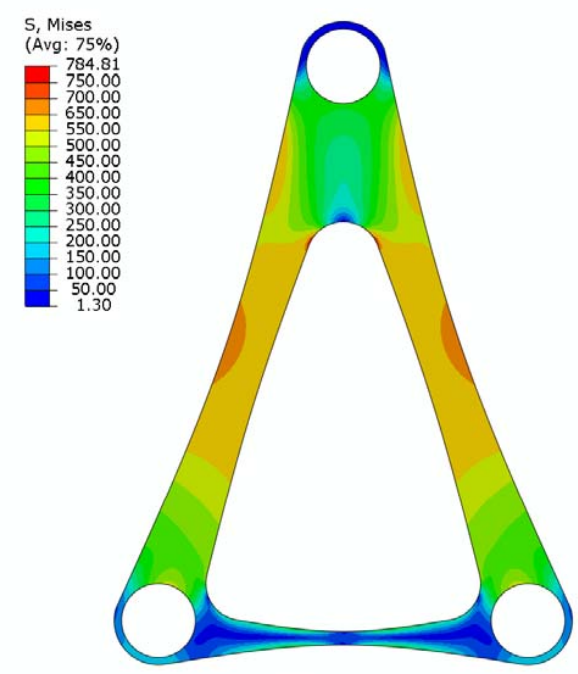

(c)

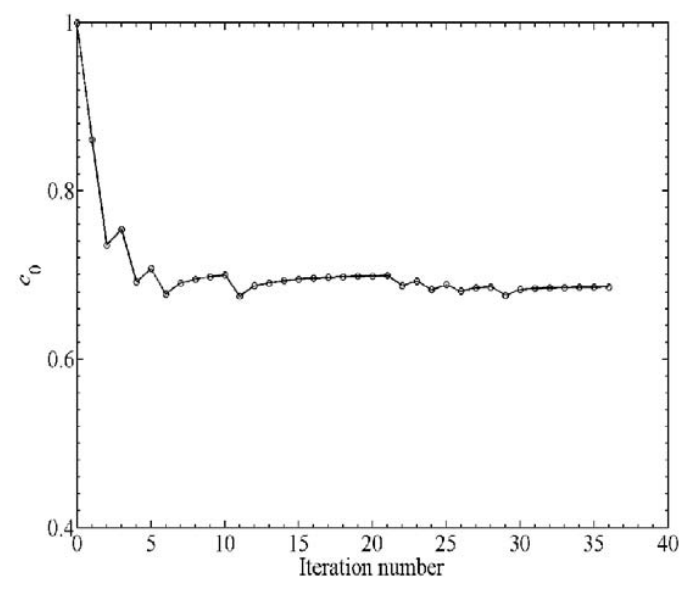

(d)

Figure 7. A three-hole bracket; (a) design parameterization; (b) von Mises stress at the initial design; (c) von Mises stress at the final design; (d) iteration history of the objective function (Example 6)

Due to their finite bounds, the random variables $X_{i}, i=1, \cdots, N$, were assumed to follow truncated Gaussian distributions with densities

$$
f_{X_{i}}\left(x_{i} ; \mathbf{d}\right)= \begin{cases}\frac{1}{\Phi\left(D_{i}\right)-\Phi\left(-D_{i}\right)} \phi\left(\frac{x_{i}-d_{i}}{\sigma_{i}}\right) & : \quad \alpha_{i} \leq x_{i} \leq \beta_{i}, \\ 0 & : \text { otherwise }\end{cases}
$$

where $\sigma_{i}=0.2$; and $\alpha_{i}=d_{i}-D_{i}$ and $\beta_{i}=d_{i}+D_{i}$ are the lower and upper bounds, respectively, of $X_{i}$. To avoid unrealistic designs, the bounds were chosen as follows: $D_{i}=2$ for all $i=1, \cdots, 9$. These conditions are consistent with the bound constraints of design variables stated in Equation 83

A multi-point single-step PDD method [22], employing univariate $(S=1)$, first-order $(m=1)$ PDD approximation of the objective and constraint functions and their design sensitivities from 
the proposed method, was employed to solve this RDO problem. Since classical orthonormal polynomials do not exist for truncated Gaussian distributions, again the Stieltjes procedure was employed to determine the measure-consistent orthonormal polynomials and corresponding Gauss quadrature formula [16]. The largest order $m^{\prime}=2$ for the Fourier polynomial expansions of the derivatives of log-density functions. The sensitivities of the first two moments of $y_{0}(\mathbf{X})$ and $y_{1}(\mathbf{X})$, required in the sequential quadratic optimization, were analytically calculated from Equations 53 and 54. Table VII summarizes the optimization results, requiring 37 design iterations and 703 FEA to attain the final optimal design with the corresponding mean shape presented in Figure 7 (c). The iteration history, depicted in Figure 7(d), indicates rapid convergence due to accurate and efficient calculation of the design sensitivities. Compared with the initial design in Figure 7(b), the overall area of the optimal design has been substantially reduced, mainly due to significant alteration of the inner boundary and moderate alteration of the outer boundary of the bracket. All nine design variables have undergone moderate to significant changes from their initial values. The optimal mass of the bracket is $0.1207 \mathrm{~kg}$ - about a $65 \%$ reduction from the initial mass of $0.3415 \mathrm{~kg}$. Due to robust design, the reduction of the mean is $65.1 \%$, whereas the standard deviation diminishes by $4.4 \%$. The smaller drop in the standard deviation is attributed to the objective function that combines both the mean and standard deviation of $y_{0}$.

Table VII. Optimization results by the univariate PDD approximation $(S=1, m=1)$ (Example 6)

\begin{tabular}{ccc}
\hline & Initial design $\left(\mathbf{d}_{0}\right)$ & Final design $\left(\tilde{\mathbf{d}}^{*}\right)$ \\
\hline$\tilde{d}_{1}^{*}, \mathrm{~mm}$ & 0 & 13.4031 \\
$\tilde{d}_{2}^{*}, \mathrm{~mm}$ & 30 & 17.0003 \\
$\tilde{d}_{3}^{*}, \mathrm{~mm}$ & 10 & 27.1802 \\
$\tilde{d}_{4}^{*}, \mathrm{~mm}$ & 40 & 30.0056 \\
$\tilde{d}_{5}^{*}, \mathrm{~mm}$ & 20 & 12.0004 \\
$\tilde{d}_{6}^{*}, \mathrm{~mm}$ & 20 & 12.0000 \\
$\tilde{d}_{7}^{*}, \mathrm{~mm}$ & 75 & 118.035 \\
$\tilde{d}_{8}^{*}, \mathrm{~mm}$ & 0 & -13.8359 \\
$\tilde{d}_{9}^{*}, \mathrm{~mm}$ & 0 & 14.9785 \\
$\tilde{c}_{0}\left(\tilde{\mathbf{d}}^{*}\right)$ & 1 & 0.6858 \\
$\tilde{c}_{1}\left(\tilde{\mathbf{d}}^{*}\right) \mathrm{MPa}$ & -433.328 & -8.084 \\
\hline
\end{tabular}

\section{CONCLUSIONS}

Three novel computational methods grounded in PDD were developed for design sensitivity analysis of high-dimensional complex systems subject to random input. The first method, capitalizing on a novel integration of PDD and score functions, provides analytical expressions of approximate design sensitivities of the first two moments that are mean-square convergent. Applied to higherorder moments, the method also estimates design sensitivities by two distinct options, depending on how the high-dimensional integrations are performed. The second method, the PDD-SPA method, integrates PDD, SPA, and score functions, leading to analytical formulae for calculating design sensitivities of probability distribution and component reliability. The third method, the PDDMCS method, also relevant to probability distribution or reliability analysis, utilizes the embedded MCS of the PDD approximation and score functions. Unlike the PDD-SPA method, however, the sensitivities in the PDD-MCS method are estimated via efficient sampling of approximate stochastic responses, thereby affording the method to address both component and system reliability problems. Furthermore, the PDD-MCS method is not influenced by any added approximations, involving calculations of the saddlepoint and higher-order moments, of the PDD-SPA method. For all three methods developed, both the statistical moments or failure probabilities and their design 
sensitivities are determined concurrently from a single stochastic analysis or simulation. Numerical results from mathematical examples corroborate fast convergence of the sensitivities of the first two moments. The same condition holds for the sensitivities of the tails of probability distributions when orthonormal polynomials are constructed consistent with the probability measure of random variables. Otherwise, the convergence properties may markedly degrade or even disappear when resorting to commonly used transformations. For calculating the sensitivities of reliability, the PDD-MCS method, especially its bivariate version, provides excellent solutions to all problems, including a 100-dimensional mathematical function, examined. In contrast, the PDD-SPA method also generates very good estimates of the sensitivities, but mostly for small to moderate uncertainties of random input. When the coefficient of variation is large, the PDD-SPA method may produce inaccurate results, suggesting a need for further improvements. Finally, a successful application on robust design optimization of a three-hole bracket demonstrates the usefulness of the methods developed.

The computational effort of the univariate PDD method varies linearly with respect to the number of random variables and, therefore, the univariate method is highly economical. In contrast, the bivariate PDD method, which generally outperforms the univariate PDD method, demands a quadratic cost scaling, making it also more expensive than the latter method. Nonetheless, both versions of the PDD method are substantially more efficient than crude MCS.

\section{ACKNOWLEDGEMENT}

The authors acknowledge financial support from the U.S. National Science Foundation under Grant No. CMMI-0969044.

\section{REFERENCES}

1. L'Ecuyer P, Perron G. On the convergence rates of IPA and FDC derivative estimators. Operations Research 1994; 42(4):643-656.

2. Glasserman P. Gradient estimation via perturbation analysis, vol. 116. Kluwer Academic Pub, 1990.

3. Rubinstein R, Shapiro A. Discrete event systems: sensitivity analysis and stochastic optimization by the score function method. Wiley series in probability and mathematical statistics, Wiley, 1993.

4. Rahman S. Stochastic sensitivity analysis by dimensional decomposition and score functions. Probabilistic Engineering Mechanics 2009; 24(3):278-287.

5. Hoeffding W. A class of statistics with asymptotically normal distributions. Annals of Mathematical Statistics 1948; 19 (3):293-325.

6. Efron B, Stein C. The jackknife estimate of variance. The Annals of Statistics 1981; 9(3):586-596.

7. Sobol I. Theorems and examples on high dimensional model representation. Reliability Engineering \& System Safety 2003; 79(2):187-193.

8. Rahman S. Approximation errors in truncated dimensional decompositions, accepted in Mathematics of Computation 2013;.

9. Bellman, R. Dynamic programming. Princeton University Press, Princeton, NJ, 1957.

10. Caflisch R, Morokoff W, Owen A. Valuation of mortgage backed securities using Brownian bridges to reduce effective dimension. Journal of Computational Finance 1997; 1:27-46.

11. Huang X, Zhang Y. Reliability-sensitivity analysis using dimension reduction methods and saddlepoint approximations. International Journal for Numerical Methods in Engineering 2012; 93(8):857-886.

12. Daniels HE. Saddlepoint approximations in statistics. The Annals of Mathematical Statistics 1954; 25(4):631-650.

13. $\mathrm{Xu} \mathrm{H}$, Rahman S. A generalized dimension-reduction method for multidimensional integration in stochastic mechanics. International Journal for Numerical Methods in Engineering 2004; 61(12):1992-2019.

14. Rahman S. Decomposition methods for structural reliability analysis revisited. Probabilistic Engineering Mechanics 2011; 26(2):357-363.

15. Rahman S. A polynomial dimensional decomposition for stochastic computing. International Journal for Numerical Methods in Engineering 2008; 76(13):2091-2116.

16. Rahman S. Extended polynomial dimensional decomposition for arbitrary probability distributions. Journal of Engineering Mechanics-ASCE 2009; 135(12):1439-1451.

17. Gautschi W. Orthogonal polynomials: computation and approximation. Numerical mathematics and scientific computation, Oxford University Press, 2004.

18. Rahman S. Statistical moments of polynomial dimensional decomposition. Journal of Engineering MechanicsASCE 2010; 136(7):923-927.

19. Lugannani R, Rice S. Saddle point approximation for the distribution of the sum of independent random variables. Advances in applied probability 1980; 12(2):475-490.

20. Yuen KV, Wang J, Au SK. Application of saddlepoint approximation in reliability analysis of dynamic systems. Earthquake Engineering and Engineering Vibration 2007; 6(4):391-400. 
21. Browder A. Mathematical analysis: an introduction. Undergraduate texts in mathematics, Springer Verlag, 1996.

22. Ren X, Rahman S. Robust design optimization by polynomial dimensional decomposition. Structural and Multidisciplinary Optimization 2012; 48(1):127-148.

23. Busbridge I. Some integrals involving Hermite polynomials. Journal of the London Mathematical Society 1948; 23:135-141.

24. Kleindienst H, Luchow A. Multiplication theorems for orthogonal polynomials. International Journal of Quantum Chemistry 1993; 48(4):239-247.

25. Oakley JE, O'Hagan A. Probabilistic sensitivity analysis of complex models: a Bayesian approach. Journal of the Royal Statistical Society: Series B (Statistical Methodology) 2004; 66(3):751-769. 